\title{
Advances in Multiple Stimuli-Responsive Drug-Delivery Systems for Cancer Therapy
}

This article was published in the following Dove Press journal:

International Journal of Nanomedicine

\author{
Ruixin Jia $\mathbb{( D}^{1, *}$ \\ Lesheng Teng (iD) ${ }^{2, *}$ \\ Lingyu Gao iD' \\ Ting Su (iD) \\ $\mathrm{Lu} \mathrm{Fu}{ }^{3}$ \\ Zhidong Qiu' \\ Ye $\mathrm{Bi}^{1,4}$ \\ 'College of Pharmacy, Changchun \\ University of Chinese Medicine, \\ Changchun, Jilin, People's Republic of \\ China; ${ }^{2}$ School of Life Science, Jilin \\ University, Changchun, Jilin, People's \\ Republic of China; ${ }^{3}$ College of Life \\ Science, Jilin Agricultural University, \\ Changchun, Jilin, People's Republic of \\ China; ${ }^{4}$ Practice Training Center, \\ Changchun University of Chinese \\ Medicine, Changchun, Jilin, People's \\ Republic of China
}

*These authors contributed equally to this work
Correspondence: $\mathrm{Ye} \mathrm{Bi}$

Practice Training Center, Changchun University of Chinese Medicine, 1035

Boshuo Road, Changchun, Jilin, People's

Republic of China

$\mathrm{Tel} / \mathrm{Fax}+86-43 \mathrm{I}-86763979$

Email biye88@outlook.com

Zhidong Qiu

College of Pharmacy, Changchun

University of Chinese Medicine, 1035

Boshuo Road, Changchun, Jilin, People's

Republic of China

$\mathrm{Tel} / \mathrm{Fax}+86-43 \mathrm{I}-86763979$

Email qzdcczy@I63.com

\begin{abstract}
Nanomedicines afford unique advantages in therapeutic intervention against tumors. However, conventional nanomedicines have failed to achieve the desired effect against cancers because of the presence of complicated physiological fluids and the tumor microenvironment. Stimuli-responsive drug-delivery systems have emerged as potential tools for advanced treatment of cancers. Versatile nano-carriers co-triggered by multiple stimuli in different levels of organisms (eg, extracorporeal, tumor tissue, cell, subcellular organelles) have aroused widespread interest because they can overcome sequential physiological and pathological barriers to deliver diverse therapeutic "payloads" to the desired targets. Furthermore, multiple stimuli-responsive drug-delivery systems (MSR-DDSs) offer a good platform for co-delivery of agents and reversing multidrug resistance. This review affords a comprehensive overview on the "landscape" of MSR-DDSs against tumors, highlights the design strategies of MSR-DDSs in recent years, discusses the putative advantage of oncotherapy or the obstacles that so far have hindered the clinical translation of MSR-DDSs.
\end{abstract} Keywords: multi-stimuli responsive nanoparticles, drug delivery, tumor microenvironment, oncotherapy

\section{Introduction}

Cancer is a major obstacle to prolong life expectancy worldwide in the $21 \mathrm{st}$ century. ${ }^{1}$ International Agency for Research on Cancer estimated that the global burden of cancer will be increasing with 21.4 million new cases and 13.2 million cancer-related deaths every year by $2030 .{ }^{2}$ Currently, surgery and chemotherapy are still the most effective means against tumor, gene therapy and immunotherapy have been increasingly utilized in cancer treatment program for the past few years. ${ }^{3}$ However, anti-tumor agents have not realized their maximum clinical therapeutic potential as non-specificity, bio-distribution, cycle stability or systemic toxicity. Drug delivery systems (DDSs) have shown a steep rise for tumor therapy application with the constantly evolving of nanotechnology, nano-carriers were widely used in oncotherapy as the characteristics of tumor target, drug-controlled release or blood long circulation. However, the conventional nanoplatforms did not achieve the expected therapeutic effect due to various barriers, which involved inconsistent of enhanced permeability and retention (EPR) effect in different tumors, scavenging effect by the mononuclear phagocytic system (MPS), premature release of agents from nanoparticles (NPs) in the blood circulation, complex tumor microenvironment (TME). TME constituted by myriad cell types, involved in tumor stem cells, tumor cells, peripheral blood vessels, cytokines, immune cells and extracellular 
matrix (ECM). ${ }^{4,5}$ Vast array of phy-chemical alterations has occurred at the cellular and tissue levels as the tumor progresses. Rapid proliferation of tumor cells induces hypoxia in tumor tissue core, hypoxia inducible factor-1 improves tumor angiogenesis. Inadequate nutrient transport changes tumor energy metabolism (Warburg effect), and extracellular metabolite lactic acid reduced $\mathrm{pH}$ of the TME. Cancer associated fibroblasts (CAFs) secreted dense ECM will further aggravate the barrier of oxygen, energy or drugs transport into the tumor core, which is associated with tumor proliferation, metastasis. ${ }^{6,7}$ There are five main dilemmas in the design of nanomedicines due to the physiological/pathological obstacles: The first dilemma is having a stable blood circulation and uptake in tumors. Cationic NPs tend to undergo cellular internalization but subsequently have a more off-target effect with rapid clearance from blood. NPs of particle size $\sim 100 \mathrm{~nm}$ are suitable for a long presence in the blood circulation but are not conducive for penetrating into the tumor core; a stromal-rich tumor only permits deep penetration by NPs of diameter $<50 \mathrm{~nm} .^{8}$ The second dilemma is NPs being able to bind the surface receptors to tumor cells. The multivalent effect prevents further cellular internalization. The third dilemma is that the EPR effect does not occur in most solid tumors (eg, bone, bladder, brain, ovaries) in humans due to a dense tumor matrix or other reasons. ${ }^{9}$ The fourth dilemma is circulation stability and the targeted release of NPs. The final dilemma is that agents with a synergistic effect are co-delivered by NPs, but they may need to be delivered into different tumor-associated cells (eg, tumor stem cells (TSCs), immune cells). Conventional NPs were unlikely to meet these complex requirements for drug delivery (Figure 1A). With ongoing advances in biomedical nanotechnology, delivery barriers of NPs have been partially solved through stimuliresponsive DDSs, called smart DDSs. Properties of smart DDSs will dramatically change upon encountering different stimuli, resulting in size shrinkage, charge reversal, ligands re-emerged or disintegration for improving tumor targeting, permeability, internalization, and cargo release in extremely proper rate. ${ }^{10}$

Uncontrolled cell proliferation, peculiar nutritional environment and metabolic patterns change of tumor tissues generated acidic $\mathrm{pH}$, elevated temperature, overexpressed proteins/enzymes, high levels of oxidationdeoxidation environment (GSH/ROS), which could be regarded as endogenous triggers. ${ }^{11,12}$ Exogenous stimuli are also used in single stimulus-responsive drug-delivery system (SSR-DDSs) designs. A manually controlled external stimulus can reduce the differences between individuals and different treatment stages to aid adaptation to complex clinical applications. The levels of triggers could be divided into four major categories. The first category is exogenous stimuli, such as light, magnetic fields, and ultrasound. Heat-triggered DDSs based on metal materials could efficiently transform energy absorbed from a magnetic field or light (usually near-infrared (NIR)) into thermal energy. The second category is NPs being able to respond to a specific tumor microenvironment (TME) to shrink its size or reverse its charge to increase the capacity of tumor permeation or cellular uptake. The third category is lowering the $\mathrm{pH}$ of endosomes/lysosomes to aid endosome escape or a "burst" release of "drug payloads". The final category is based on overexpressed proteins/enzymes, high concentration of adenosine triphosphate and chemical reduction in the cytoplasm to accelerate the release of the drug payload or reach nuclear/ mitochondrial targets.

An ideal NPs requires overcoming sequential physiological/pathological obstacles for effectively delivery agents against tumor, including blood circulation stability, tumor accumulation, tumor penetration, synergistic effect on different tumor-associated cells, cancer cell internalization and lysosome escape. ${ }^{13}$ SSR-DDSs can overcome one of the biological barriers, but other obstacles may hinder effective delivery of the drug. SSR-DDSs are not sufficiently accurate due to the off-target effects induced by normal cells/tissues with a similar stimulating environment. $^{14,15}$ Endogenous SSR-DDSs rely on the abnormal environment in diseased tissue, and they need to know the target location in advance to carry out efficacious treatment. For instance, photo stimuli-responsive NPs are often affected by the light penetration of different carcinomas in situ. ${ }^{16}$ Also, a stimulus may not be sufficiently robust to trigger a response; the extracellular $\mathrm{pH}$ in the TME may have only a slight difference from that of normal tissues, which can result in a slow/no response from NPs. ${ }^{17}$

Multiple triggers co-existing may better control the spatial location of agents to achieve accuracy and flexibility. ${ }^{18}$ Multiple stimuli-responsive drug-delivery systems (MSRDDSs) were introduced for precise treatment of tumors, and are based on a mixture of endogenous/exogenous stimuli. Compared with SSR-DDSs, MSR-DDSs can integrate several activatable strategies into a single nanoplatform with multiple functions to overcome a series of continuous 


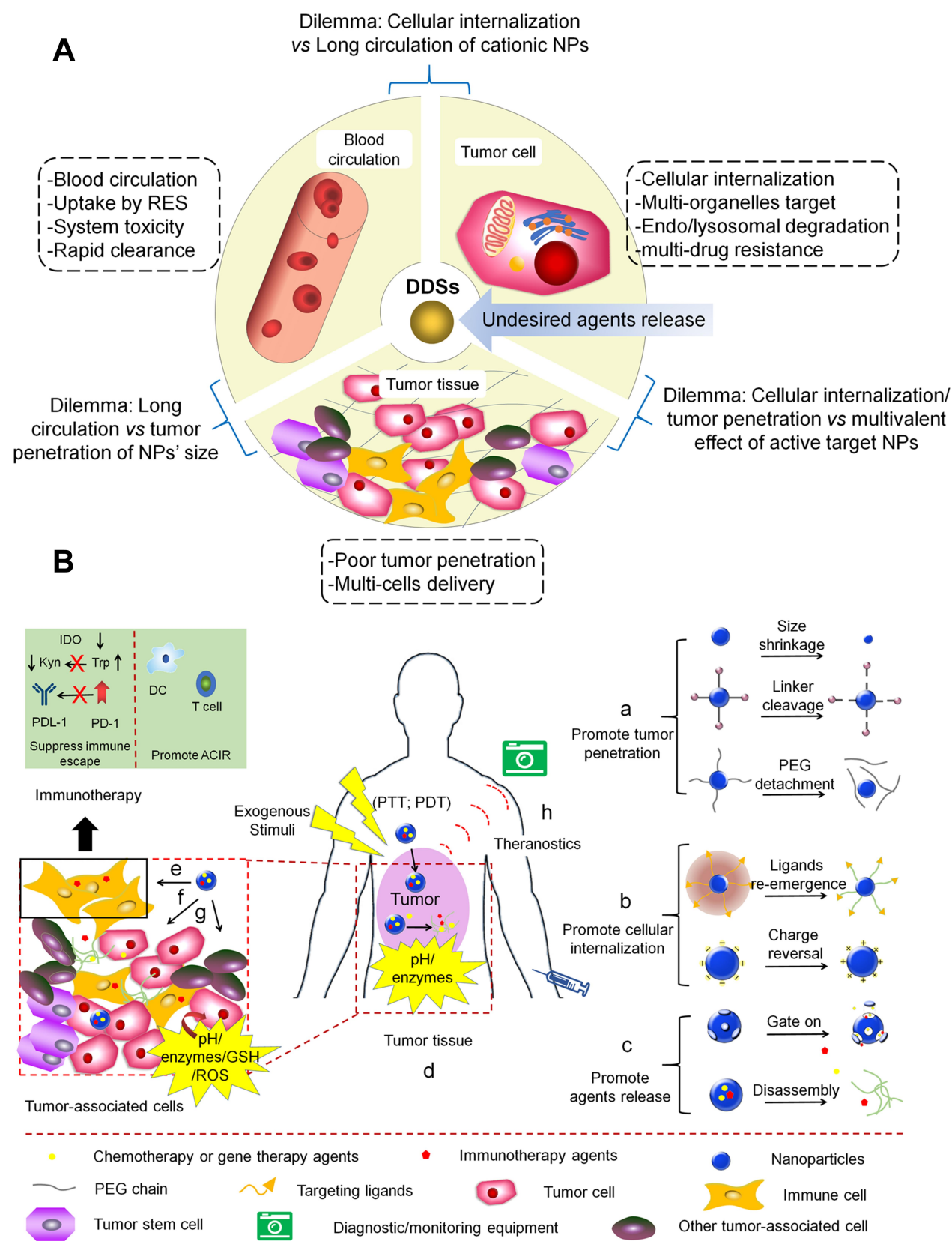

Figure I MSR-DDSs for tumor precise treatment. (A) The design dilemmas of nanomedicine as the compound physiological/pathological barriers. (B) MSR-DDSs overcome a series of barriers and dilemmas through integrating different single stimulus response strategies. (a-c) NPs response the stimulus to realize function by changes their structure; (d) MSR-DDSs target tumor tissue by responding endogenous or exogenous stimuli; (e-h) MSR-DDSs further response stimuli to realize immune cells, tumor cells, other tumor-associated cells effective delivery, or theranostic.

Abbreviations: IDO, indoleamine 2,3-dioxygenase; Kyn, I-kynurenine; Trp, I-tryptophan; PD-I, programmed cell death-I; PDL-I, programmed cell death-ligand I; PTT, photothermal therapy; PDT, photodynamic therapy.

physiological/pathological barriers and dilemmas of simple nano-systems by responding to different levels of stimuli one-by-one or simultaneously to achieve advanced delivery of drugs. MSR-DDSs can response to multiple stimuli for multistage site-specific drug delivery to eventually realize the highest therapeutic efficacy. MSR-DDSs can break down the barriers as the beginning of the burgeoning field precision cancer nanomedicine, which also provide a promising platform for combination of cytotoxic nanomedicine, tumor "theranostics" (diagnosis/therapy plus multi-mode imaging) and immunotherapy to realize more efficacious "personalized" therapy (Figure 1B). ${ }^{12}$ Revolutionary immunotherapy has changed the therapeutic manipulation of several advanced-stage tumors. However, the positive rate of patients responding to immunotherapies is still limited in solid tumors as delivery barriers. 
MSR-DDSs can deliver agents into TEM to regulate immunosuppressive cells, regulate immunosuppressive factors and enhance immunogenicity against tumor by spatiotemporally controlling immune responses, which provides an innovative way of tumor immunotherapy. Combinations of different stimuli will satisfy the different requirements when multiple stimuli-responsive strategies are integrated into a nano-system. Thus, the sequence of different stimuli must be considered carefully. The design complexity of MSR-DDSs is also one of obstacles must be addressed, the multi-stimuli may not respond one-by-one or simultaneously as expected, which hinders precisely control the spatio-temporal delivery of agents.

This review highlights the design strategies of MSRDDSs with different stimulation levels and critically discuss their advantages and limitations, which might offer new insights into the potential for tumor treatment.

\section{MSR-DDSs Triggered by Combined Stimuli in Different Physiological Environments MSR-DDSs Responsive to Exogenous and Tumor-Tissue Triggers}

Exogenous stimuli possess the advantages of simple handling, highly controllable treatment time and location, so they were extensively used for achieving accurate agents'controlled delivery. ${ }^{17}$ Meanwhile, MSR-DDSs can accurately locate and diagnose tumor by magnetic resonance or optical imaging property. ${ }^{19}$ Integration of diagnosis and treatment with NPs mediated by exogenous stimuli will be introduced in subsequent chapters. Exogenous stimuli could be regarded as a "switch" to seal the pores of NPs with little leakage of drug and a burst release at targeted sites by triggers, such as a magnetic field, light or heat. ${ }^{20,21}$ However, dual exogenous trigger-responsive DDSs are not conducive to personalized treatment because of the individual differences of patients, different stages of disease development, and the cellular heterogeneity of tumor cells. DDSs triggered by exogenous factors and the TME could enable application of diverse therapeutic methods aimed at different sites/cells with deepening of tumor tissue. An endogenous TME stimulus could further rectify the deviations of individual differences (Figure 2).

The smart NPs can be decomposed into several individual parts with different characterization by stimuli to target different tumor-associated cells with sequentially or simultaneously agents release for collaborating treatment and avoiding competition. Tumor tissuetriggers responding is a promising tactics for tumor depth delivery. Lei et al reported a stimuli-responsive NPs like "Cluster Bomb" could synergistically kill tumor cells at different depths with combination chemotherapy and photothermal therapy (PTT). ${ }^{22}$ The acid-labile benzoicimine bond is unstable as the linker between "bomblets" and "dispense" when NPs reaching the acidic TME, "Cluster Bomb" rapidly divided into two parts: mesoporous silica NPs (MSNs, as dispense) loaded with DOX concentrated on the surface of tumor tissues and endocytosed by tumor cells in the vicinity of blood vessels; tLyP1-modified tungsten disulfide quantum dots $\left(\mathrm{WS}_{2}-\mathrm{HP}\right.$, as bomblets) with smaller size possessed strong tumor penetration ability, which is used as medium for PTT by transforming NIR to kill internal malignant cells which are far from the blood vessels (Figure $3 \mathrm{~A}-\mathrm{C}$ ). ${ }^{23}$ Through the combination of in-depth chemotherapy and PTT, tumor inhibition rate of "Cluster Bomb" reached 93\%, and the relative tumor volume, excised tumor weigh significantly reduced compared with about $70 \%$ inhibition rates of DOX@MSN-WS2-RP (Figure 3D and E).

Biomacromolecules (eg, proteins, RNA, DNA) are sensitive to enzymatic degradation. They must be bound to special molecules or subcellular structures within cells to act. This strategy requires NPs to help agents escape endosomes into the cytosol. In general, nucleic acids with a negative charge often combine with cationic polymers through electrostatic interactions to improve their stability during systemic delivery. ${ }^{24}$ However, release of nucleic acids at the target site is a challenge because of strong binding forces. Advanced inorganic materials called "heterostructures" (HSs) are arranged in a clear geometric shape. They are formed by sharing the interface of $\geq 2$ inorganic NPs' domains to integrate the functional subdomain of NPs with different drugs into one carrier. Kakwere et al used $\mathrm{Au}-\mathrm{Fe}_{\mathrm{x}} \mathrm{O}_{\mathrm{y}}$ dimers as a template to prepare "smart" inorganic HSs with excellent heating performance in magnetic fields, and $\mathrm{pH} /$ temperature-responsive polymers were attached to $\mathrm{HSs}$ of $\mathrm{Au}-\mathrm{Fe}_{\mathrm{x}} \mathrm{O}_{\mathrm{y}}$ for clinical applications. $^{25,26}$ Iron oxides of HSs can be used as magnetic resonance imaging (MRI) agents and thermal media for magnetism-induced hyperthermia or heat triggers; the existence of gold NPs improves the heat dissipation of the iron-oxide domain under an alternating magnetic field. ${ }^{20}$ A heat-responsive polymer, poly ( $\mathrm{N}$-isopropyl acrylamide) (PNIPAAM), has been connected selectively to the gold domain by a gold mercaptan bond to load Nile Blue dye. 


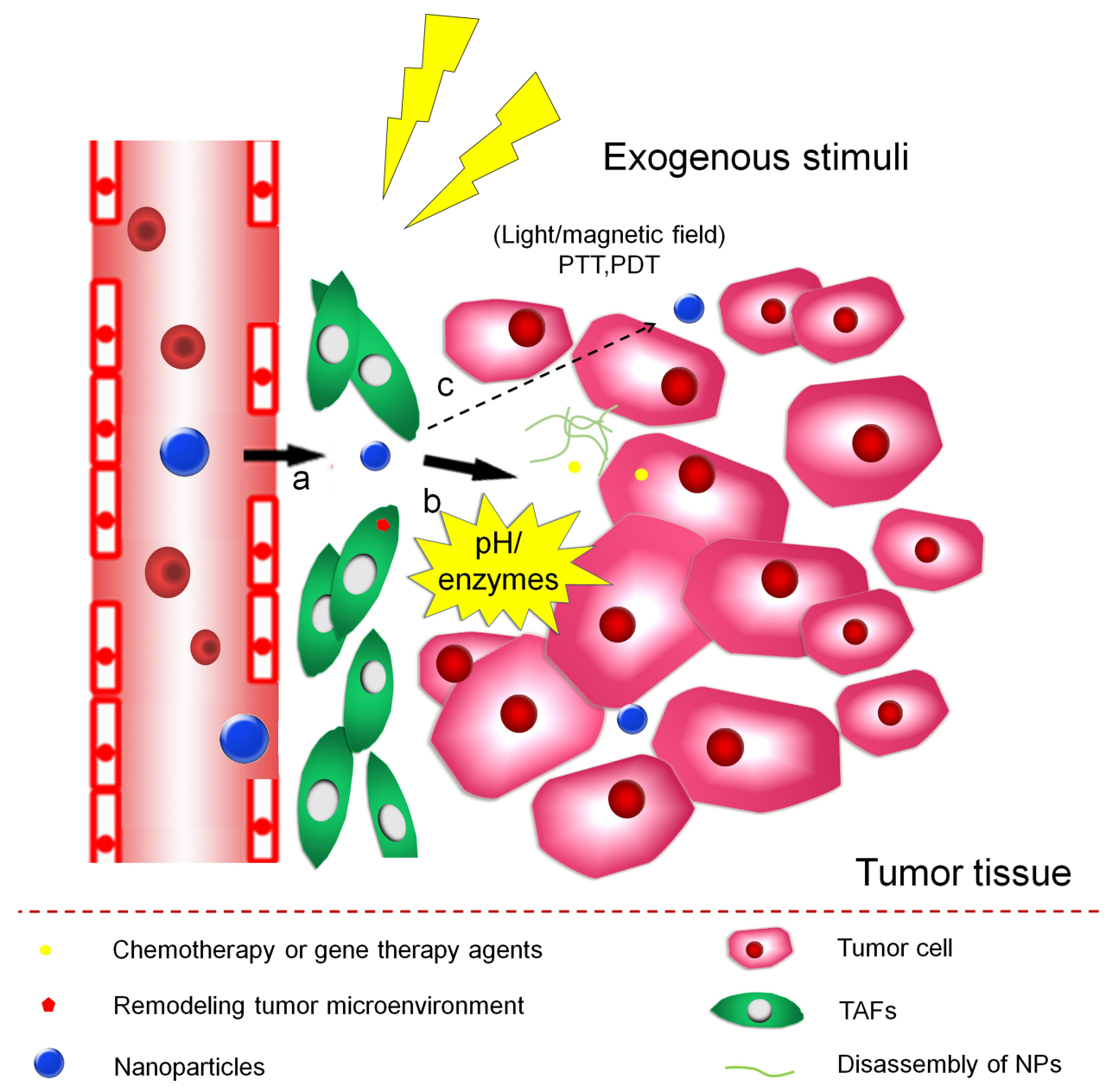

Figure 2 MSR-DDSs trigger by exogenous factors and TEM stimuli for (a) size shrinkage and (b) accelerated drug release, (c) tumor tissue penetration. Abbreviation: TAFs, tumor-associated fibroblasts.

In addition, a pH-responsive polymer, poly (dimethylaminoethyl acrylate) (PDMAEA), has been attached to the surface of iron oxide through a catechol-modified initiator, which can realize the delivery of anti-green fluorescent protein-small interfering RNA (GFP-siRNA). Upon HSs reaching the acidic microenvironment of the tumor, PDMAEA undergoes hydrolysis to reverse the potential from positive to negative to release siRNA by polymer cleavage and charge repulsion. ${ }^{27}$ Conversely, if the temperature is lower than the low critical solution temperature (LCST; $43^{\circ} \mathrm{C}$ ), PNIPAAM in HSs is soluble in water and can undergo a conformational change from a coil to a globule to trigger payload release while heating above the LCST by an alternating magnetic field. ${ }^{28}$ Anti-GFPsiRNA-loaded Au-FexOy HSs were shown to silence GFP by $40 \%$ without toxicity, whereas free siRNA or HSs without a payload showed little downregulation. Moreover, HSs not only provided a good platform for drug co-delivery but also exhibited potential as contrast agents in MRI applications. Additional MSR-DDSs responsive to exogenous and tumor-tissue triggers for delivering antitumor agents are listed in Table 1.

\section{MSR-DDSs Responsive to Exogenous and Cellular/Subcellular Triggers}

Exogenous stimuli could combine with cellular/subcellular-triggers to further facilitate intracellular agents release or effective endosomal escape (size shrinkage, charge reversal, ligands re-emergence) while favorable cellular internalization to implement complex multifunctions (Figure 4). This section focuses on the design concept of NPs triggered by exogenous and cellular/subcellular stimuli combination for effectively delivering chemotherapeutic, genetic therapeutics.

Molecules that can coat the surface of NPs to make them more hydrophilic can provide a "stealth" property for 

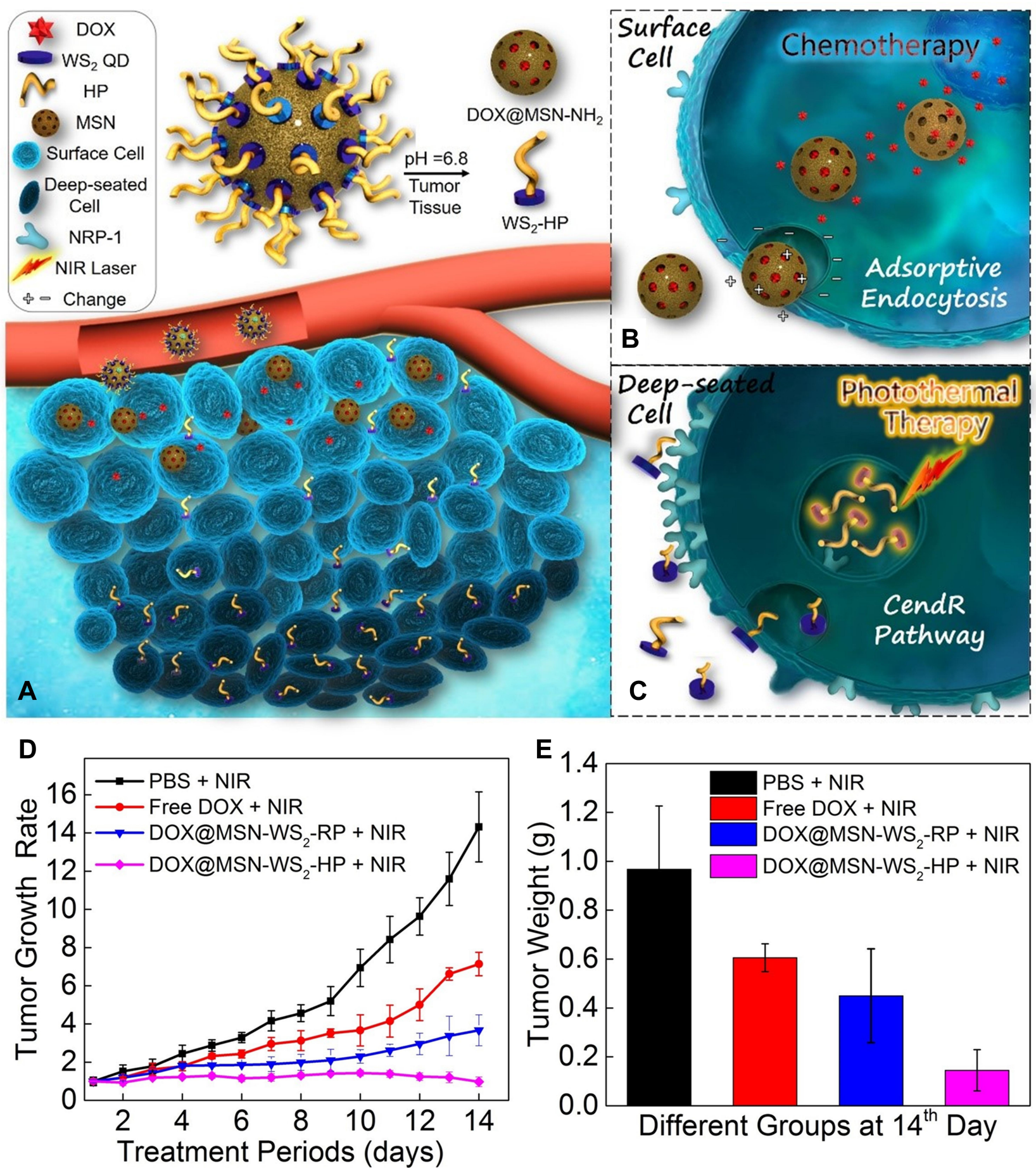

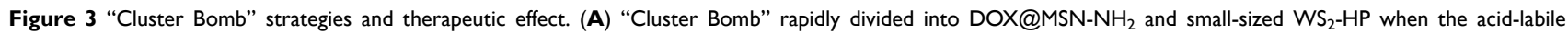
nanoplatform reaching the acidic TME; (B) DOX loaded MSNs concentrated on the surface of tumor cells and endocytosed; (C) WS 2 -HP with smaller size possessed strong tumor penetration ability, which be used as medium for PTT; (D) tumor growth curves of the tumor-bearing mice; (E) excised tumor weight.

Notes: Reprinted from Lei Q, Wang SB, Hu JJ, et al. Stimuli-Responsive "Cluster Bomb" for Programmed Tumor Therapy. ACS Nano. 20I7; I (7):720I-72I4. Copyright (2017), with permission from ACS. ${ }^{22}$

NPs in the systemic circulation. Such molecules include polyethylene glycol (PEG) and hyaluronic acid. PEGmodified NPs improve passive tumor targets by prolonging the time in the blood stream, but the outer layer of
PEG also hinders further cellular uptake of NPs ("PEG dilemma"). ${ }^{29}$ It is desirable that PEG detaches from NPs only when NPs accumulate in tumor tissue to improve tumor penetration and cellular uptake without 


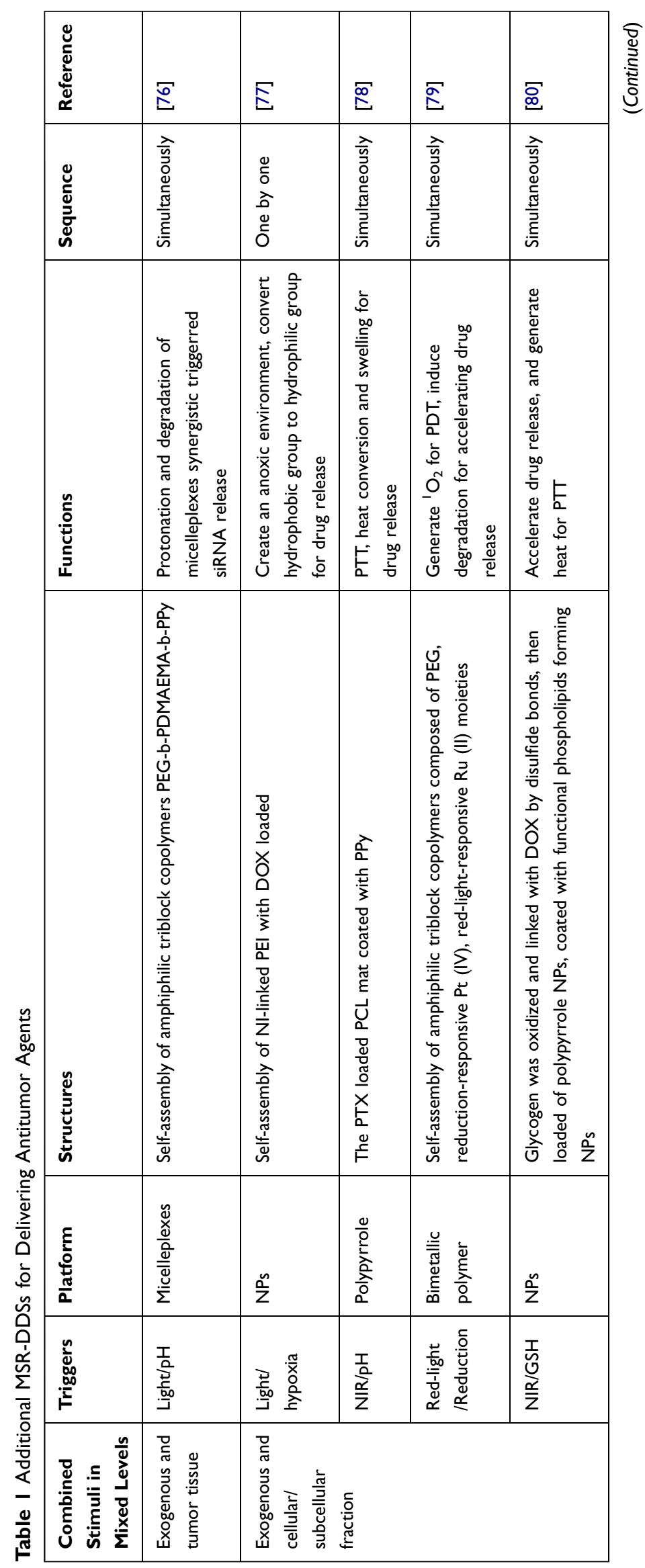




\begin{tabular}{|c|c|c|c|c|c|}
\hline 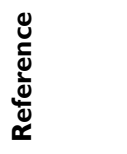 & $\overline{\bar{\alpha}}$ & $\underset{\infty}{\widetilde{D}}$ & $\stackrel{\widetilde{m}}{\underline{\infty}}$ & $\underset{\Phi}{F}$ & $\stackrel{\sqrt[5]{\infty}}{\mathscr{\infty}}$ \\
\hline 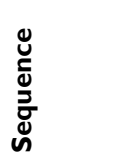 & $\begin{array}{l}\check{\Xi} \\
0 \\
0 \\
\searrow \\
0\end{array}$ & 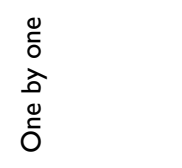 & 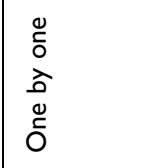 & $\begin{array}{l}\searrow \\
0 \\
0 \\
0 \\
\searrow \\
0\end{array}$ & $\begin{array}{l}\searrow \\
\check{0} \\
\widehat{\partial} \\
\stackrel{0}{0} \\
0\end{array}$ \\
\hline 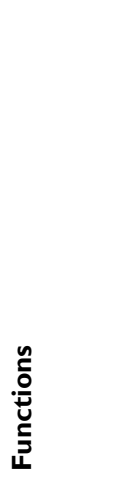 & 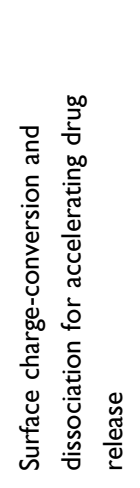 & 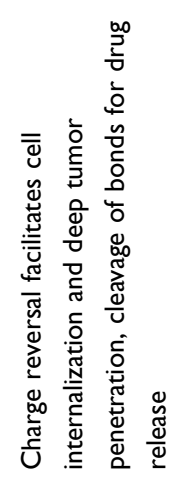 & 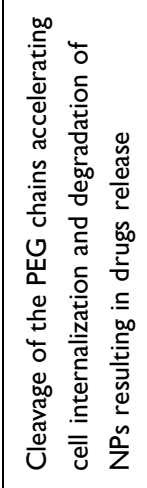 & 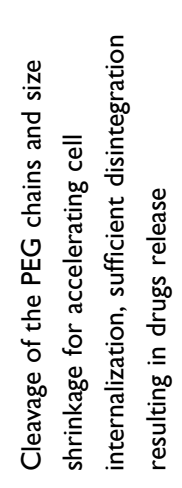 & 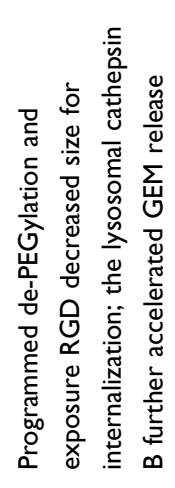 \\
\hline 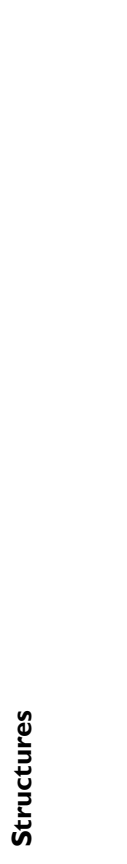 & 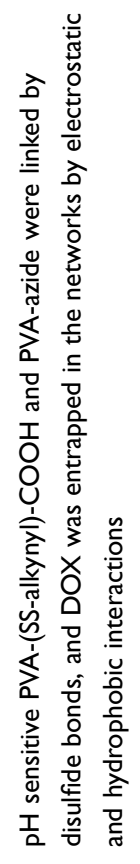 & 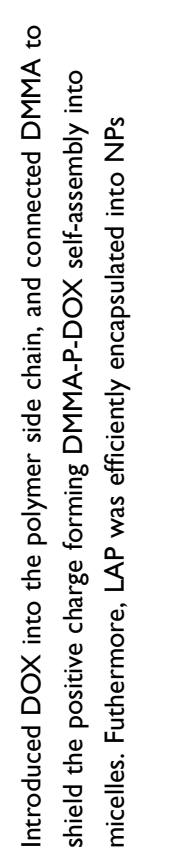 & 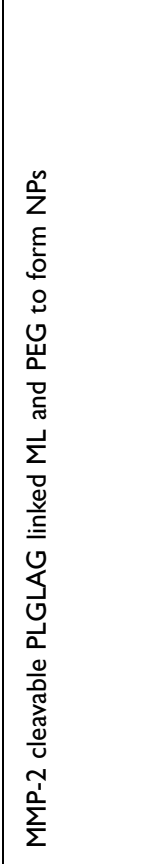 & 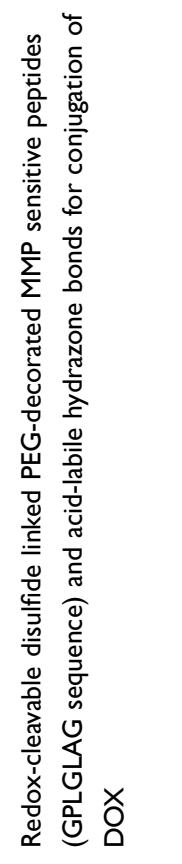 & 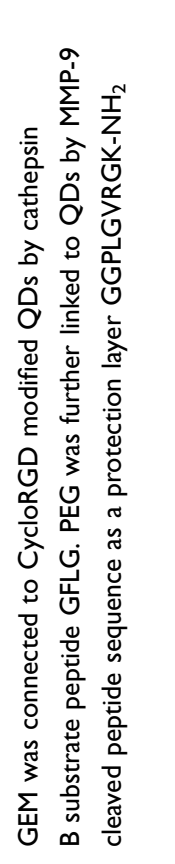 \\
\hline $\begin{array}{l}\varepsilon \\
\frac{\varepsilon}{0} \\
\frac{\pi}{2} \\
\frac{\pi}{2}\end{array}$ & $\begin{array}{l}\bar{d} \\
\text { on } \\
\stackrel{\circ}{c} \\
\tilde{Z}\end{array}$ & $\begin{array}{l}\frac{\mathscr{\varrho}}{\bar{\Xi}} \\
\stackrel{\breve{\Sigma}}{\Sigma}\end{array}$ & $\stackrel{n}{z}$ & 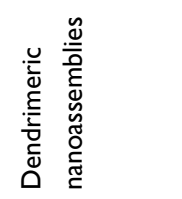 & 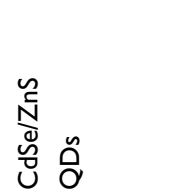 \\
\hline 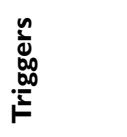 & 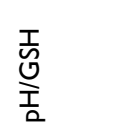 & 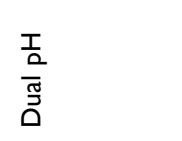 & 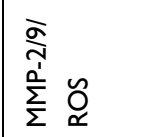 & 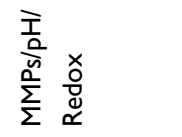 & 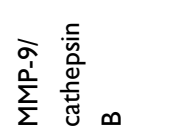 \\
\hline 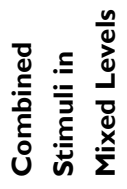 & \multicolumn{5}{|c|}{ 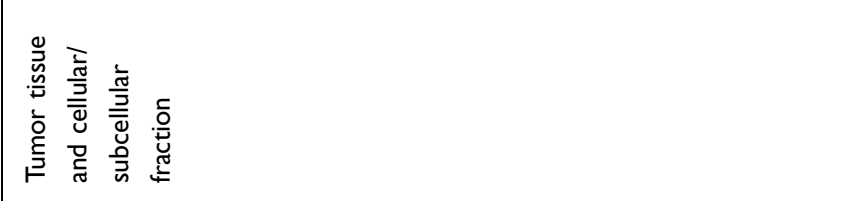 } \\
\hline
\end{tabular}




\begin{tabular}{|c|c|c|c|c|c|c|c|c|c|c|}
\hline 递 & $\underset{\infty}{\Gamma}$ & 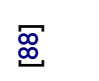 & $\underset{\underset{\infty}{\infty}}{\bar{\alpha}}$ & $\bar{\Sigma}$ & $\bar{\Xi}$ & $\Xi$ & $\bar{\sigma}$ & F & $\stackrel{5}{2}$ & 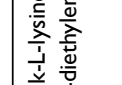 \\
\hline 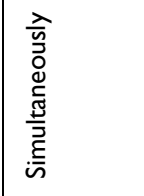 & 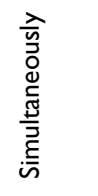 & 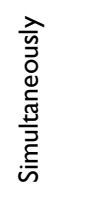 & 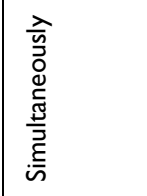 & 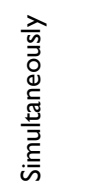 & 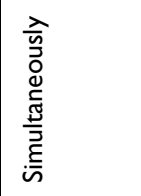 & 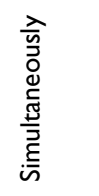 & $\begin{array}{l}0 \\
\check{0} \\
\text { Oे } \\
0 \\
0 \\
0\end{array}$ & $\begin{array}{l}0 \\
\check{0} \\
\widehat{\partial} \\
0 \\
0 \\
0\end{array}$ & $\begin{array}{l}0 \\
\check{0} \\
\widehat{\partial} \\
0 \\
0 \\
0\end{array}$ & 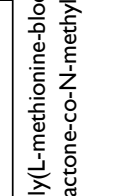 \\
\hline 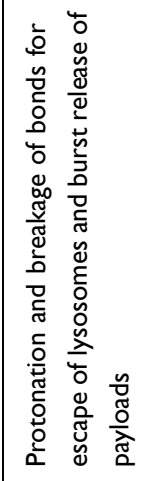 & 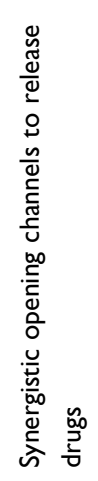 & 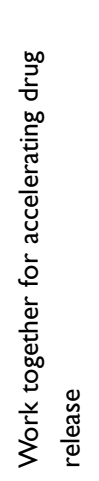 & 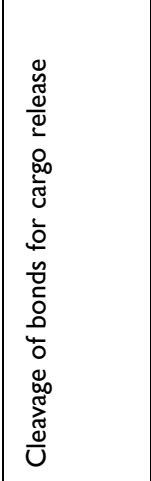 & 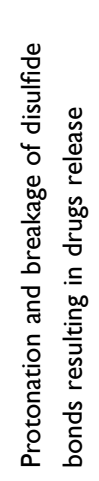 & 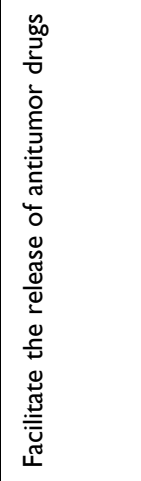 & 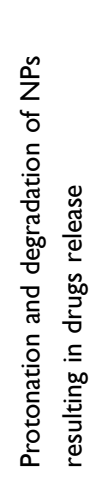 & 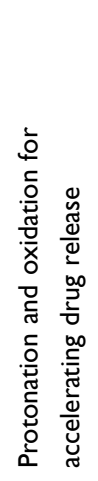 & 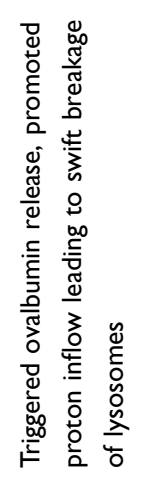 & 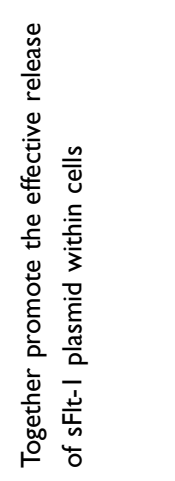 & 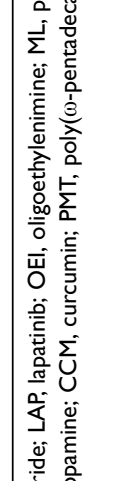 \\
\hline 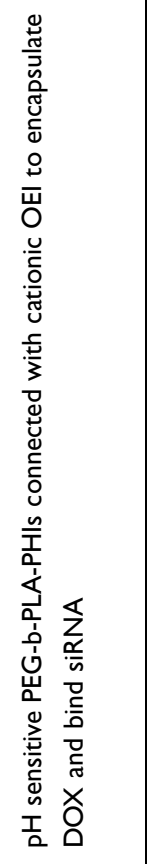 & 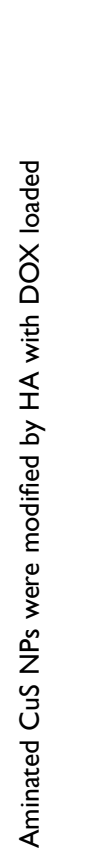 & 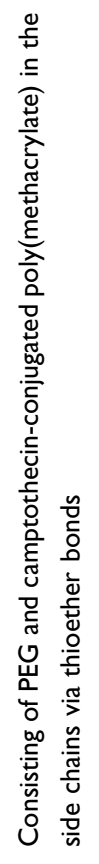 & 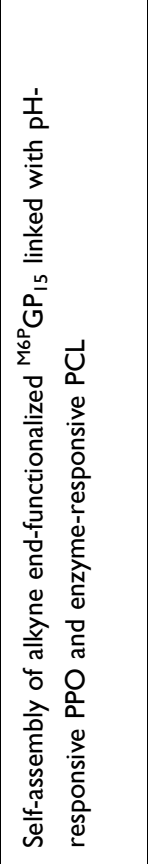 & 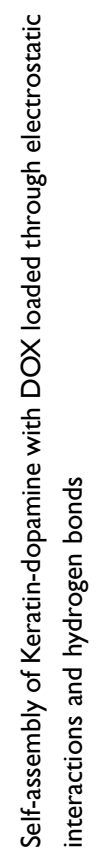 & 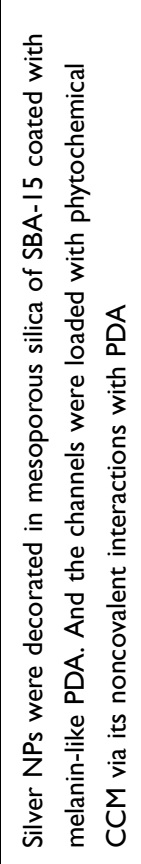 & 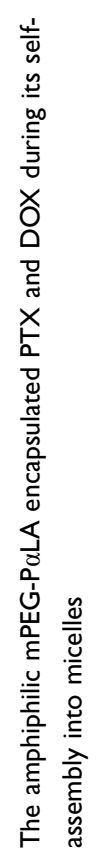 & 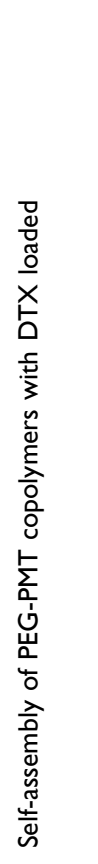 & 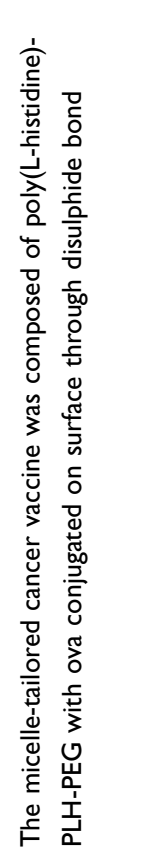 & 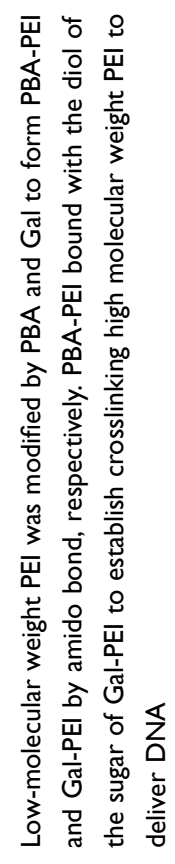 & 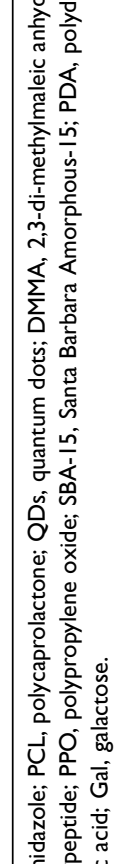 \\
\hline $\begin{array}{l}\frac{x}{0} \\
\frac{0}{0} \\
\frac{0}{0}\end{array}$ & 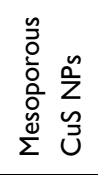 & 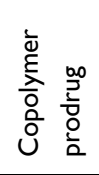 & $\begin{array}{l}0_{2}^{n} \\
0 \\
0 \\
\vdots \\
\vdots\end{array}$ & $\overline{\mathbf{z}}$ & 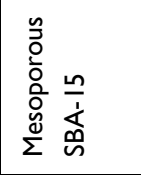 & 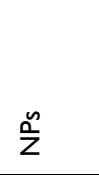 & $\begin{array}{l}\frac{\mathscr{y}}{\overline{\underline{\nu}}} \\
\frac{\stackrel{\underline{\Sigma}}{\Sigma}}{}\end{array}$ & 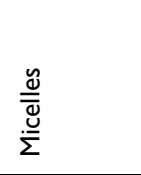 & $\overline{\mathbf{z}}$ & \\
\hline 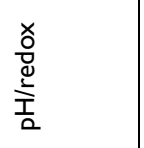 & $\begin{array}{l}\text { 焉 } \\
\text { 崖 }\end{array}$ & 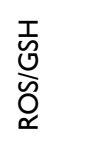 & 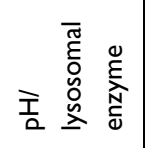 & 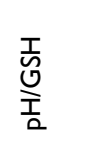 & 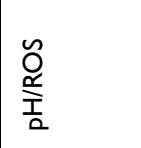 & 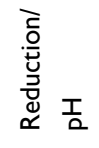 & 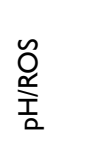 & 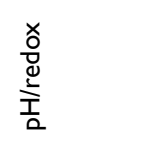 & $\begin{array}{l}\frac{E}{\frac{K}{I}} \\
\frac{1}{a}\end{array}$ & . \\
\hline 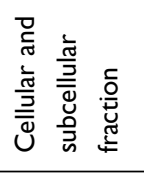 & & & & & & & & & & \\
\hline
\end{tabular}



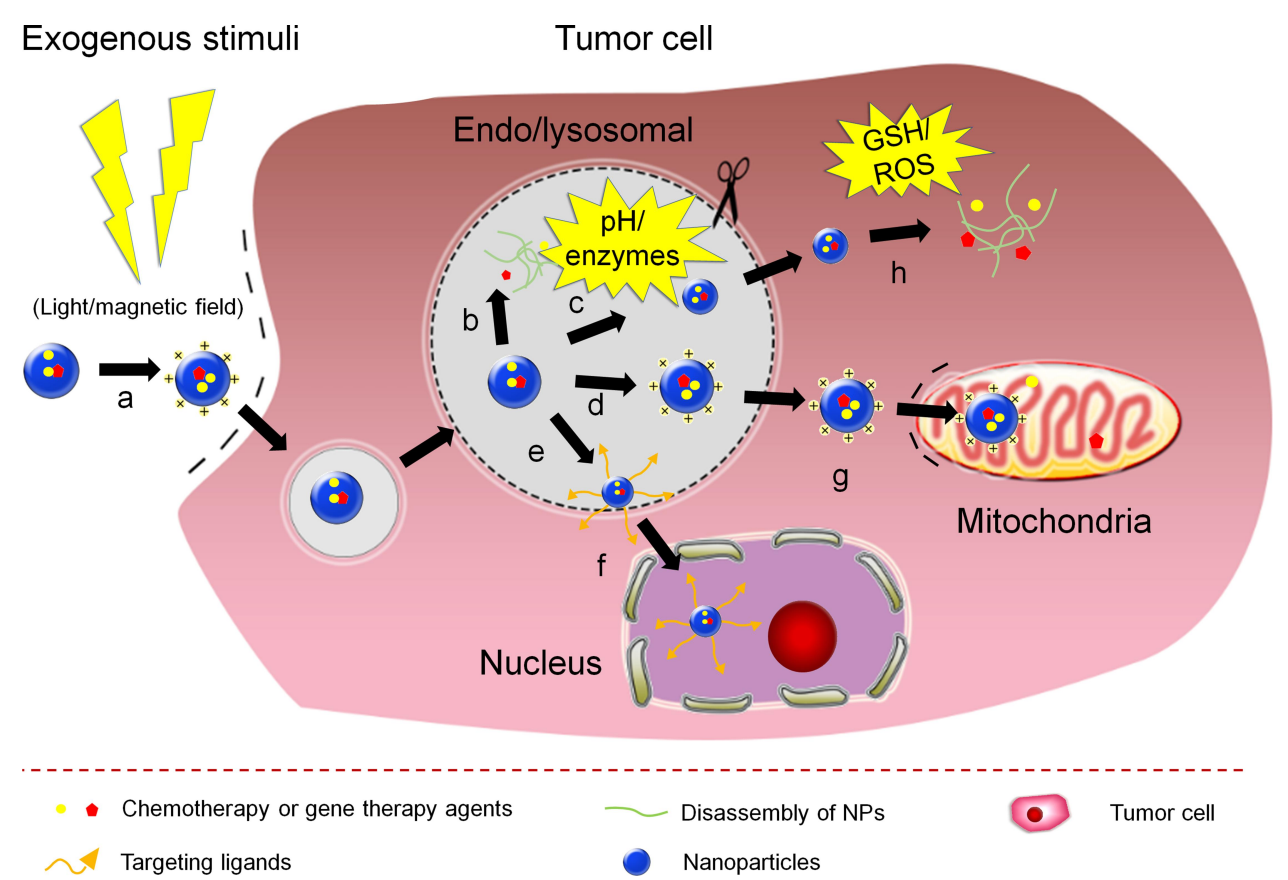

Figure 4 MSR-DDSs can promote cellular internalization through (a) charge reversal or ligands re-emergence under exogenous stimuli; Subcellular-stimuli of lysosomal environment trigger (b) NPs disintegration or effective endosomal escape (c, size shrinkage; $d$, charge reversal; e, ligands re-emergence) for further ( $f$ ) nuclear localization and (g) mitochondrial targeting, and cellular-stimuli of the high level of GSH/ROS can induce $(\mathrm{h})$ breakdown of NPs for agents release.

eliciting off-target effects. A "Trojan horse" strategy is used widely in MSR-DDSs designs for tumor-specific stimulation triggers by PEGylation/dePEGylation of NPs on blood circulation or target-sites. A high density of PEG-modified NPs results in poor penetration of tumors and suboptimal anti-tumor efficacy because of the larger particle size and hydrophilia. Exogenous and cellular/subcellular triggers could realize tumor-targeted drug delivery in a temporal and spatial-specific fashion. Zhou et al reported that NIR light/pH-responsive micelles (iPUDN) could co-deliver doxorubicin (DOX) and "up-conversion" NPs (UCNPs) against breast cancer. ${ }^{30}$ DOX and UCNPs were enveloped by amphiphilic mixed micelles comprising the polymers PEG-Nbz-PAE-Nbz-PEG and iRGD-PAEiRGD. The target ligands of iRGD were shielded by PEG to reduce nonspecific uptake in the blood circulation. The chemical groups of $\mathrm{Nbz}$ and PAE were sensitive to UV and acidic $\mathrm{pH}$, respectively. Furthermore, the embedded UCNPs could convert NIR light into UV light to remove PEG from iPUDN to the tumor target. In addition, the smaller particle size of iPUDN improved the tumor penetration of NPs. The subsequent acidic $\mathrm{pH}$ within tumor cells promoted iPUDN disassembly through PAE degradation to release DOX. iPUDN disassembly to release DOX was enhanced from $\sim 27 \%$ to $\sim 80 \%$ at $\mathrm{pH} 5.0$ upon activation by NIR light. iPUDN with NIR light potently suppressed tumor growth and showed high accumulation in tumors with a significantly long circulation time in blood compared with that using UCNPs. Exogenous stimuli could induce dePEGylation of NPs to improve cellular uptake markedly. Cellular stimuli could trigger size shrinkage of NPs to improve deep penetration into tumors.

Most stimuli-responsive nano-carriers are constructed from chemically synthesized materials, which may limit their biocompatibility. Ideal vector itself can afford "ondemand" drug release ability according to exogenous or endogenous stimuli and possess inherent anti-tumor activity. Bilirubin exhibits superior anticancer activity and the ability of converting from hydrophobic to hydrophilic under ROS/light, so it can also be used as nano-carrier against tumor. ${ }^{31}$ Under the irradiation of appropriate wavelength, bilirubin will undergo photoisomerization (through the breaking of intramolecular hydrogen bonds) and convert into water-soluble photoisomers to accelerate payload release. ${ }^{32}$ The bilirubin NPs (BRNPs) loaded with DOX through $\pi-\pi$ interactions and hydrogen bonds rapidly decomposed under light irradiation to release $80 \%$ of DOX within 5 mins. On the other hand, ROS exposure led to the oxidation of bilirubin to biliverdin, which also significantly increased water solubility of carriers to contribute agents release. ${ }^{33}$ Notably, BRNPs not 
only could be used as drug carriers but also anti-tumor drugs due to the inherent activity. The BRNPs alone can achieve a tumor inhibition rate of $38.1 \%$, which retain free bilirubin intrinsic anti-tumor effect. As combined drugdelivery carriers and nano-medicine, BRNPs can be simply prepared on a large-scale production with great application prospect.

Cationic polymers or liposomes possess high transfection efficiency but inevitably have serious toxicity. Cationic polymers attached onto the surface of inorganic NPs are a feasible method to reduce toxic effects. ${ }^{21}$ In general, MSNs are considered to be suitable polymer supports for gene/combination therapy because the surface can provide support sites for polymer modification as "gatekeepers" to control the switching of MSN channels under stimuli for reducing untargeted leakage of drugs. Meanwhile, the porous channels provide more space for drug loading. ${ }^{34}$ Zhou et al used UV light-crosslinked and pH-de-crosslinked coumarin groups as gatekeepers to control the release of loaded agents. ${ }^{21}$ Cationic polymers can also be connected to the surface of MSNs by stimuliresponsive bonds for binding to genetic drugs, which act as gatekeepers of the MSNs' channels to stably encapsulate the cargo with reduced leakage of the drug. For instance, Zhou and coworkers provided a vector for DOX and B-cell lymphoma (Bcl)-2 siRNA co-delivery to induce the apoptosis of breast-cancer cells based on polyminecopolymerized lysine (PEI-PLL)-modified MSNs. ${ }^{34}$ The disulfide bonds between PEI-PLL and MSN could be cleaved by glutathione (GSH) to achieve effective release of DOX by "open" gatekeepers. Meanwhile, the protonation of amine groups in PEI-PLL increased the electrostatic repulsion between DOX molecules and polymers to accelerate drug release, and the mutual coulombic repulsion of ionized amino groups led to MSN swelling for further DOX release. ${ }^{35,36}$ The release of DOX at $\mathrm{pH} 5.0$, 7.4 was increased from $34 \%, 11 \%$ to $58 \%, 45 \%$ after addition of GSH $(10 \mathrm{mM})$, which revealed that acidic TME/lysosome and reducing environment could be together used in NPs designs for rapid agents release. Meanwhile, co-delivery of DOX and Bcl-2 siRNA increased apoptosis by $14.37 \%$ as compared with that using only DOX-loaded MSNs.

Gold nanorods (GNRs) have also garnered considerable attention because of their unique ability of high-efficiency photothermal conversion. This platform is suitable for remotely controlled release of gene agents based on light triggers. ${ }^{37}$ According to the aspect ratio of GNRs, the surface plasmon resonance of GNRs can regulate incident light (from visible-light to NIR regions) at the nanoscale for conversion to heat. In particular, the "water window" (650-$1100 \mathrm{~nm}$ ) of NIR light has low absorption in skin and tissue and possesses maximum efficiency for photochemical conversion. ${ }^{38}$ Wang and coworkers constructed NIR/GSHresponsive gold NPs to deliver p65-short hairpin shRNA (an agent that works against nuclear factor-kappa B) for treating metastatic cancer. ${ }^{39}$ The tumor-targeting peptides of RGD were conjugated to DSPEI (a short PEI was used to absorb gene-therapy agents by electrostatic adsorption) to synthesize RGD-PEG-DSPEI through PEG spacers. GNRs were surface-functionalized with RGD-PEG-DSPEI segments by gold mercaptan bonds for a GSH response. A 2-nm polymer layer was formed onto the GNR surface with a particle size of $\sim 50 \mathrm{~nm}$ (RDG/shRNA). A NIR laser and the "proton-sponge" effect of DSPEI helped NPs to escape from endosomes/lysosomes after effective uptake by tumor cells. ${ }^{38}$ Meanwhile, NIR-induced transfer of "hot" electrons and a high concentration of GSH within tumor cells reduced the electrostatic adsorption of shRNAs and broke disulfide bonds to trigger shRNA release into the cytosol. This action improved gene-silencing efficiency to enhance the anti-metastatic effect against 4T1 breast-cancer cells. NPs accumulated effectively into tumor tissue $(12.3 \%$ of the injected dose $6 \mathrm{~h}$ after intravenous injection). RDG/shRNA treatment upon irradiation with a NIR laser reduced tumor volume by $83.5 \%$ compared with that in the control group, and inhibited pulmonary metastasis significantly. Exogenous stimuli can help smart NPs to achieve an optimal therapeutic effect. However, exogenous stimuli are weakened with an increasing depth of the target site and with inferior "personalization" to the target cell. Auxiliary equipment is needed during treatment, which hinders the development of the NPs response to exogenous stimuli. Additional MSR-DDSs triggered by exogenous and cellular/subcellular stimuli for delivering antitumor agents are listed in Table 1.

\section{MSR-DDSs Responsive to Tumor Tissue and Cellular/Subcellular Triggers}

The delivery and distribution of agents are hampered markedly by the heterogeneity of tumor tissue (eg, proliferation of tumor stromal cells, crosslinking of the extracellular matrix, vascular abnormalities in the tumor, or abnormal increases in interstitial fluid pressure). Especially in solid tumors, the efficiency of NPs delivery can be improved by changing 
the size, configuration or charge of NPs to overcome barriers in tumor tissue. ${ }^{17,40,41}$ The TME conditions of low $\mathrm{pH}$ (6.5-7.2) or overexpressed enzymes in the extracellular environment can induce size shrinkage by de-PEGylation or NP decomposition to reach different tumor depths after smart NPs accumulate in tumor tissues through the EPR effect or ligands. NPs with ionizable groups undergo charge reversal by protonation in an acidic TME to facilitate cell internalization. In addition, MSR-DDSs can release agents in tumor tissues to act on different cells (eg, tumor cells, tumorassociated fibroblasts, immune cells or immunogenic cells). MSR-DDSs loaded with multiple agents have a synergistic role for superior anti-tumor activity. Conversely, NPs triggered by cellular/subcellular stimuli have a burst-type release of intracellular payloads. Further protonation of endosomes/lysosomes can also endow pH-responsive NPs with a greater positive charge, which contributes to satisfactory mitochondrial targeting because a strong negative charge is present in the mitochondria matrix. ${ }^{42}$ NPs that are responsive to tumor tissue and cellular/subcellular stimuli can be applied to multistage delivery of agents and a combination of different therapeutic methods (Figure 5).

The particle size of NPs can affect their biological distribution and accumulation in tumor tissues significantly. Conventional NPs (of size $\sim 100 \mathrm{~nm}$ ) barely penetrate into deep tumor tissue due to the obstruction proffered by dense tumor stroma. NPs of size $<30 \mathrm{~nm}$ have a promising capacity of interstitial transport and tumor penetration. However, small NPs have poor tumorspecific distribution with a short half-life. ${ }^{43}$ Therefore, a two-step strategy is adopted for better delivery of drugs. A conventional initial size of NPs is conducive to long circulation in the blood stream, selective extravasation, and accumulation in tumors. The shrinkage in size triggered by stimuli in the TME further improves penetration when NPs enter tumor tissue. Yu and coworkers synthesized a size-shrinkable vehicle for DOX delivery to balance the contradiction of circulation in the blood stream and tumor penetration through a temperature/pH/ redox response. $^{44}$ Mixed micelles composed of poly (N-isopropylacrylamide) (PNIPAM), 2-hydroxyethylmethacrylate (HEMA), methacrylic acid (MAA) and $\mathrm{N}$, $\mathrm{N}^{\prime}$-bis(acryloyl) cystamine (BACy) were crosslinked by a disulfide bond through distillation-precipitation polymerization. The destruction of $\mathrm{pH}$-responsive MMA and thermosensitive PNIPAM caused the volume of the carrier to decrease to diffuse further into the tumor under the stimulation of abnormal $\mathrm{pH}$ and temperature of tumor tissue. Furthermore, BACy containing disulfide bonds introduced an additional reduction responsiveness (response to a high intracellular level of GSH) to accelerate DOX release from micelles after tumor-cell internalization. Under the effect of three types of triggers, smart NPs could simultaneously obtain tumor accumulation, tumor penetration, and controlled release of loaded cargo. In a simulated TME, the drug was released rapidly

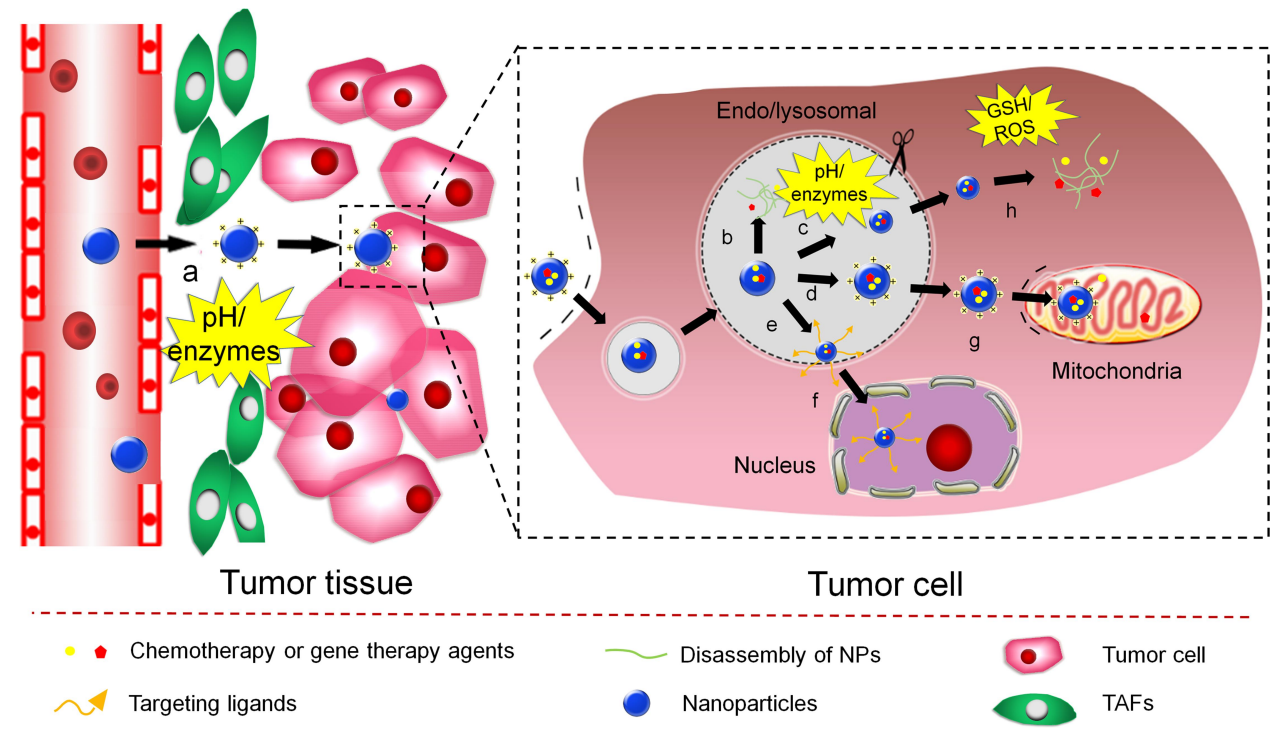

Figure 5 MSR-DDSs responsive to tumor tissue and cellular/subcellular triggers for (a) effective cellular internalization, (b and $h$ ) agents release, (c-e) endosomal escape, (f) nuclear localization and $(\mathrm{g})$ mitochondrial targeting. 
after $8 \mathrm{~h}$, and percent release reached $80 \%$ after $24 \mathrm{~h}$. Using a single stimulus, the drug was released at a low rate.

The other strategy apart from variable size is using sensitive chemical bonds to conjugate two sizes of NPs to obtain deep delivery into tumors through stimulation, but without changing the main structure of individual NPs. Ruan and collaborators reported 19-nm DOX-tethered gold NPs (AuNPs) conjugated with shrinkable gelatin NPs to prepare a $187-\mathrm{nm}$ nanocarrier (G-AuNPs-DOXPEG). ${ }^{43}$ DOX was linked to AuNPs by hydrazone bonds. AuNPs were conjugated with gelatin by an MMP-2 responsive peptide sequence. G-AuNPs-DOX-PEG was degraded by extracellular overexpressed MMP-2 in the tumor matrix to separate AuNPs to reduce the particle size. Subsequently, the hydrazone bonds were cleaved to release DOX once AuNPs were within the intracellular acidic environment. The penetrating percentage (fluorescence intensity of inner region/edge region) of G-AuNPsDOX-PEG at $140-\mu \mathrm{m}$ sections of $4 \mathrm{~T} 1$ tumor spheroids was improved more than twofold after 12-h pre-treatment with MMP-2. Distribution of G-AuNPs-DOX-PEG showed greater accumulation in the edge region of 4T1 tumor slices, and showed only a slight decrease in the deep region of the tumor (5-mm from the edge). Varying the particle size can be used for the delivery of agents deep into tumors.

Apart from particle size, the surface charge of NPs is also a vital parameter that affects the penetration and targeting of tumor cells, and cellular uptake. The effect of charge on NP delivery is also contradictory. NPs with positive charge tend to aggregate with plasma proteins, be absorbed by normal cells with a negatively charged membrane, and be recognized and eliminated by the reticuloendothelial system. ${ }^{45}$ Thus, MSR-DDSs that can switch their surface charge, which carry a negative/neutral charge during circulation, or can reverse to a positive charge in the TME for cellular internalization are required. Mo et al designed charge-reversible zwitterionic oligopeptide liposomes ( $\mathrm{HHG} 2 \mathrm{C}_{18}-\mathrm{L}$ ) comprising soy phosphatidylcholine and 1,5-dioctadecyl-glutamyl,2-histidyl-hex-ahydrobenzoic acid $\left(\mathrm{HHG}_{2} \mathrm{C}_{18}\right)$ to target mitochondria with multiplestage $\mathrm{pH}$ responsiveness. The synthetic lipid $\mathrm{HHG} 2 \mathrm{C}_{18}$ contained two stearyl alkane chains as a hydrophobic block, a pH-cleavable group (hexahydro benzamide) as well as glutamic acid and histidine as a hydrophilic block. $^{46}$ The potential of liposomes was reversed to positive to promote cellular uptake in the TME. The proton- sponge effect and hydrolysis of $\mathrm{HHG} 2 \mathrm{C}_{18}$ produced a stronger positive surface charge after endocytosis into endosomes/lysosomes. The zeta potential of $\mathrm{HHG} 2 \mathrm{C}_{18}-\mathrm{L}$ at $\mathrm{pH} 7.4$ was only $-20 \mathrm{mV}$, whereas it was $+22 \mathrm{mV}$ and $+40 \mathrm{mV}$ at $\mathrm{pH} 5.5$ and $\mathrm{pH} 4.5$, respectively. HHG2C18-L helped to target mitochondria by electrostatic actions, and could kill tumor cells by destroying their energy sources.

The nucleus is another pharmacological target for many first-line chemotherapy agents. The size of NPs becomes a limiting factor for nucleus delivery due to the nucleopore threshold $(<9 \mathrm{~nm}) .{ }^{47}$ NPs can be modified with a nuclear localization sequence (NLS) of peptides to promote targeted delivery to the nucleus. The NLS induces NPs to become moderately larger than the nucleopore threshold and aids nuclear entry. ${ }^{48,49}$ Nevertheless, a cationic NLS makes the distribution of NPs in tissues nonspecific and promotes rapid clearance after systemic administration. Li and coworkers designed polymer nanovesicles (PNVs) with multiple-stage changes in size/ charge to achieve higher accumulation in nuclei. ${ }^{50}$ Each N-(2-hydroxypropyl) methacrylamide (HPMA)-based PNV comprised two copolymers of opposite charge: an anionic 2,3-dimethylmaleic anhydride (DMA)-decorated HPMA conjugate (P-DMA) and cationic P-Dox R8NLS containing intracellularly detachable subgroups (IDSs). DOX linked to a secondary targeting group, R8NLS, was used to modify HPMA to form P-DoxR8NLS by formation of hydrazone bonds. The acidic nature of the TME induced reversal of the P-DMA charge, which resulted in PNV disintegration into a linear conjugate for first-stage size reduction (from $55 \mathrm{~nm}$ to $10 \mathrm{~nm}$ ) and exposed $\mathrm{R} 8$ ligands with enhanced internalization. Furthermore, cleavage of hydrazone bonds in acidic lysosomes led to IDS destruction, and a second-stage size shrinkage and R8NLS re-emergence promoted nuclear-targeted DOX release. This strategy further supported the carrier size to guarantee nuclear entry, and showed 4.5-fold higher nuclear accumulation than that observed using DOX-loaded HPMA.

Charge reversals triggered by endogenous stimuli can synergistically enhance NPs' accumulation in tumors with particle-size shrinkage. Cisplatin is a first-line drug used against lung cancer. It forms platinum (Pt)-DNA adducts to interfere with DNA replication. ${ }^{51}$ Nevertheless, tumor resistance severely hinders cisplatin application, which is caused mainly by cisplatin inactivation, low intake of cisplatin, efflux of cisplatin and DNA repair. ${ }^{45}$ Gemcitabine (GEM) is used against multiple solid tumors because it can inhibit DNA repair. Therefore, 
a combination of GEM and Pt is an important strategy for overcoming drug resistance. Liu et al constructed a hollow MSN (HMSN)-based multifunctional nanocarrier to deliver a GEM- and cisplatin-conjugated poly(amidoamine) dendrimer (PAMAM-Pt). ${ }^{52}$ The contradiction between the blood circulation and cell internalization of NPs was addressed by a charge-reversal strategy. HMSNs were covered by a dimethylmaleic anhydride-modified chitosan oligosaccharide (CS-DMA) "stealth layer" to prevent nonspecific protein absorption and MPS recognition for long circulation in the blood stream. The multifunctional nanocarrier was decomposed in HMSN@GEM-CS (120 nm) and PAMAM-Pt $(5 \mathrm{~nm})$ by an acidic TME. A lower $\mathrm{pH}$ also mediated the protonation of CS-DMA to lead chargereversal for improving cellular uptake of large-size HMSN@GEM-CS. Thus, the isolated PAMAM-Pt of small size and positive charge could diffuse into tumor parenchyma. Upon entering the intracellular cytosol after endocytosis, dendritic PAMAM-Pt molecules released active cisplatin to kill deep-lying tumor tissue cells by overexpressed GSH. The drug concentration of cisplatin was enhanced $\geq 4$.4-fold by the multifunctional nanocarrier compared with that using free cisplatin or PAMAM-Pt. HMSN@GEM-CS (DMA)/PAMAM-Pt extended the lifespan of $50 \%$ of mice by $>2$ months. In conclusion, charge/ size-changeable nano-vectors exhibited superior performance in tumor penetration, cellular uptake, and intracellular transport through a stimuli-responsive strategy.

MSR-DDSs can work cooperatively on different tumor-related cells. Conventional anticancer agents often aim to kill tumor cells. The existence of TSCs contributes to DNA repair and tumorigenesis. Combined inhibition of tumor cells and TSCs could be a viable strategy. Liu et al prepared a tumor-related stimuli-activated nanogel (aNG) using in situ interfacial polymerization for co-delivery of cytotoxic ribonuclease A (RNase A) and anti-TSC doxycycline (Doc) after overcoming sequential physiological barriers. $^{53}$ aNG $(\sim 100 \mathrm{~nm})$ was established as a coreshell polymer by hierarchical assembly, RNase A was first encapsulated in a reduction-responsive single proteinbased nanocapsule to form R-rNC of particle size $<10 \mathrm{~nm}$ and a positive surface potential for tumor perfusion and rapid release. Then, a polymeric core was formed by acrylamide, a cationic monomer N-(3-aminopropyl) methacrylamide, azide-decorated monomer $\left(\mathrm{AAm}-\mathrm{N}_{3}\right)$, and an acid-cleavable glycerol dimethacrylate (GDA) as crosslinker to load R-rNC and Doc. The polymeric core was shielded by dibenzocyclooctyne-modified hyaluronic acid (DBCO-HA) conjugated to the azide group of AAm- $\mathrm{N}_{3}$ to improve the circulation and targeting ability of aNG (Figure 6A). aNG was collapsed directly in an acidic condition and divided into two parts (Doc and R-rNC), which had a satisfactory effect against tumor cells and TSCs for tumor eradication, respectively (Figure 6A). ${ }^{54}$ The small-sized R-rNC with a positive surface charge can be absorbed readily by tumor cells and biodegraded to release RNase A, whereas the small molecule Doc eradicated TSCs by inhibiting mitochondrial biogenesis. ${ }^{55-58}$ aNG could actively target tumor tissue and diffuse deep into tumors. These co-delivered agents synergistically reduced the average cluster of differentiation (CD) $133^{+}$subpopulation (surface marker of TSCs) from $10.5 \%$ to $0.69 \%$, and reduced $73.6 \%$ of tumor weight compared with that in control cells. Tumor inhibitory of ratio of Doc/R-rNC/nNG (76\%) was significantly higher than no morphological change of the non-degradable Doc/ R-rNC/nNG (low than 40\%) (Figure 6B and C). Taken together, those data suggest that hybrid NPs can achieve collaborative therapy on MSR-DDS platforms by targeting tumor cells and TSCs, respectively.

The toxicity of foreign material is also an area of consideration for MSR-DDSs. Peptides with good biocompatibility can also be used as carriers to deliver agents but the toxicity of the synthetic material must be considered very carefully. Li and collaborators reported peptide-based NPs that could release a therapeutic peptide $(\mathrm{Pe})$ and Curcumin (Cur) sequentially in response to MMP, a low $\mathrm{pH}$ of lysosomes, and intracellular $\mathrm{GSH}^{59}$ Interestingly, the Pe could be used not only as a drug to antagonize breast cancer by interfering with the transmembrane domain of ErbB-2 but could also be a ligand to recognize tumor cells specifically. ${ }^{60}$ Partially hydrolyzed $\alpha$ lactalbumin fragments were self-assembled into a peptosome (PS) to load Cur, and crosslinked with endogenous cysteines by disulfide bonds. Through maleimide (MAL), the PS/Cur complex was conjugated further with Pm (GPLGVRGC; a peptide can be effectively cleaved by MMP)-modified Pe to form a biomimetic peptide body: $\mathrm{PePm} / \mathrm{PSs} / \mathrm{Cur}$. The linker Pm on PS could be hydrolyzed by MMP, thereby leaving Pe to bind and inhibit breast cancer cells by recognizing and interfering with ErbB-2 receptors. Simultaneously, the positive charge of residual MAL-PS/Cur promoted the tumor-cell internalization of NPs and induced subsequent lysosome escape by the proton-sponge effect. Upon cell entry, PS/Cur was susceptible to a high concentration of GSH to release Cur into the 

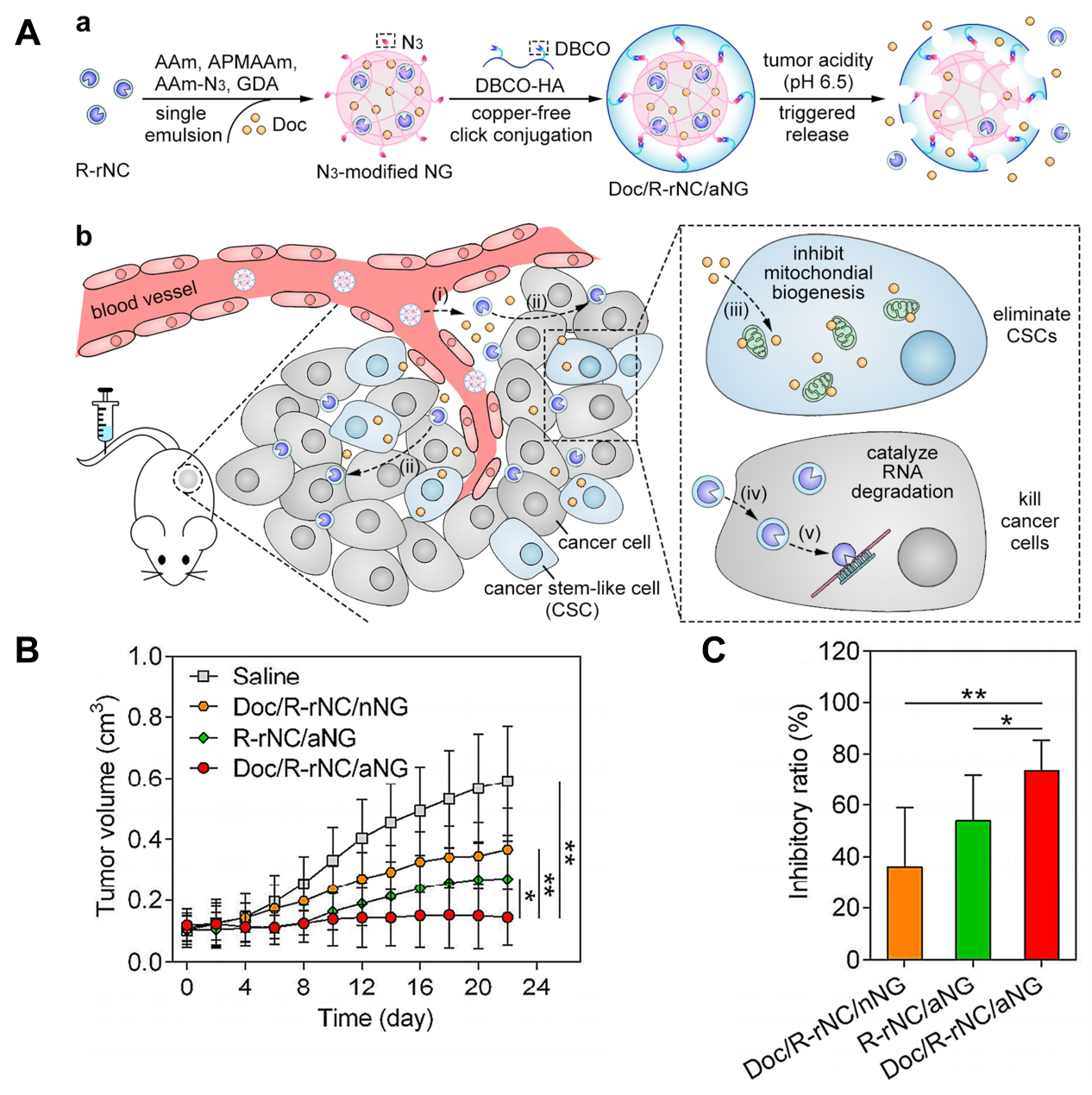

Figure 6 The design strategy of Doc/R-rNC/aNG. (A) Assembly and disassembly of Doc/R-rNC/aNG; Doc/R-rNC/aNG sequentially delivery R-rNC and Doc to cancer cells and CSCs; (B) in vivo antitumor performance of Doc/R-rNC/aNG, relative tumor volume; (C) inhibitory ratio of the tumor growth. (*P < 0.05 , **P < $0.0 \mathrm{I})$.

Notes: Reprinted from Liu M, Shen S, Wen D, et al. Hierarchical Nanoassemblies-Assisted Combinational Delivery of Cytotoxic Protein and Antibiotic for Cancer Treatment. Nano Lett. 2018;18(4):2294-2303. Copyright (2018), with permission from ACS. ${ }^{53}$

cytoplasm by reduction. The fluorescence intensity of $\mathrm{PePm} / \mathrm{PS}$ was approximately threefold that of naked PS upon replacement of Cur with the hydrophobic dye $\mathrm{Cy} 7$, which revealed exceptional accumulation in tumors.

Amino cationic polymers like poly (ethyleneimine) (PEI), polylysine (PLL), poly (2-(dimethylamino)) ethyl methacrylate (PDMAEMA) and chitosan were widely used as gene delivery carriers. ${ }^{61}$ PEI has good transfection efficiency by destroying endosomes through proton-sponge mechanism. ${ }^{62}$ PEI with molecular weight of $25 \mathrm{kDa}$ is considered to be the "gold standard" for gene delivery, it shows high transfection efficiency and low toxic. ${ }^{63} \mathrm{Xu}$ et al reported a dual-ligands targeting, stimuli-responsive gene delivery platform based on PEI to promote pTRAIL DNA and monensin (the death receptor sensitizer) codelivery. ${ }^{64,65}$ Low molecular weight PEI (LMW-PEI) was crosslinked with sulfhydryl cyclodextrin by disulfide bond to form bio-reducible core to deliver DNA and monensin. Meanwhile, the nucleus complex was modified by RGD (tumor homing peptide) and $\gamma$-PGA (Poly- $\gamma$-Glu acid) to mediate endocytosis and partially shield the excessive positive charge on the surface of NPs during circulation. In addition, $\gamma$-PGA can also specifically target tumor cells by binding to the tumor-associated gamma-glutamyl transpeptidase. The shedding of the surface coating would fall off with particle size reducing since $\gamma$-PGA is $\mathrm{pH}$-sensitive in TME, following accelerated cellular internalization. The decomposition of disulfide by reduction condition triggered the leakage of loaded degradable biochemical reagent within tumor cells. The apoptosis rate induced by $\gamma$-PGA /PEI/pTRAIL NPs was $82.9 \%$ almost twice times of PEI/ pTRAIL NPs. In vivo experiments showed that doubleligands targeted MSR-DDSs could significantly suppress tumor growth with inhibition rate of $85.7 \%$, whereas the 
PEI/pTRAIL NPs were merely $15.2 \%$. Compared with plasmid DNA, siRNA has lower charge density and molecular weight, so high doses of cationic lipids or polymers are needed to increase the loading capacity and stability of siRNA, which leads to additional severe cytotoxicity at healthy non-target tissues and the failure of RNA-induced silencing complex (RISC) activation with insufficient release of siRNA in the cytoplasm as the enhanced affinity between siRNA and carriers. ${ }^{66}$ In order to decrease systemic toxicity without reducing delivery efficiency, Saw et al imitated an amphipathic cationic lipid using two hydrophobic stearic acid tails modified Mitoxantrone (SAMTO). Mitoxantrone (MTO) is an anticancer drug with cationic properties, which can absorb siRNA (PLK1) to form a prodrug complex (SA-MTO/siRNA) by electrostatic binding. ${ }^{67,68}$ SA-MTO/siRNA was encapsulated in $\mathrm{pH}-$ responsive methoxyl-poly (ethylene glycol)-b-poly(2-(pentamethyleneimino)ethyl methacrylate) (Meo-PEG - $b$-PPMEMA) for tumor delivery. Protonation of PPMEMA segments induced by acidic TME that can lead to NPs disassembled to pre-expose ionized SA-MTO /siRNA complexes with small size for promoting tumor permeability and cytoplasmic transport. Furthermore, overexpressed esterase in tumor cells can hydrolyze the amphiphilic structure of SA-MTO prodrug, resulting in second-stage release of prodrug complexes and finally suppressing tumor growth through MTO-mediated chemotherapy and siRNA-mediated gene silence. NPs with $\mathrm{N} / \mathrm{P}$ ratio of 15 revealed moderate $\zeta$ potential and smaller particle size, and Meo-PEG- $b$-PPMEMA also did not show significant toxicity (about 90\% cells viability even at a polymer concentration of $100 \mathrm{mg} / \mathrm{L})$. This stimuliresponsive hybrid nanoplatform integrated of polymer, prodrug-siRNA complex has a huge potential in anti-tumor agents' multistage delivery and combination therapy. Additional MSR-DDSs triggered by tumor tissue and cellular/subcellular fraction for delivering antitumor agents are listed in Table 1.

\section{MSR-DDSs Responsive to Cellular and Subcellular-Triggers}

The changes of TME are not very dramatic, even under if pathological conditions. Tumor tissue-triggers are not stable enough, the low $\mathrm{pH}$ or up-regulation of enzymes in normal tissues may lead to off-target effects. In another case, the weak acid of tumor tissue may cause the response to be incomplete or un-triggered. Increasing the penetration of tumor tissue and the ability of cellular internalization both lay the foundation for the targeted drug release of NPs within tumor cells. The advantage of cellular and subcellular-triggers responsive NPs in accelerating loaded-agents release has received widespread attention with superior efficiency and facilitating endosomal escape. Due to vacuolar proton ATPase-mediated proton influx, the $\mathrm{pH}$ value of endosome and lysosome is lower than 6.0, NPs with ionizable groups (including amine, acyl sulfonamide, and imidazole) produce proton sponge effect to increase osmotic pressure in the inner compartment, eventually, realizing endosomal escape with the disruption of the plasma membrane. ${ }^{69,70}$ Meanwhile, the low $\mathrm{pH}$ value of endo/lysosomal is the most widely used to achieve subcellulartargeted drug release by adding acid-sensitive bonds (such as hydrazone bonds) or protonated groups. However, acidic lysosome is common in normal cells not specific to tumor cells, so NPs triggered by lysosomes are needed to combine with other tumor cellular-specific stimuli to prevent offtarget effects (Figure 7). Cellular-triggered delivery systems relied on the reductive environment in tumor cells have gained much more attention, disulfide bond with the characteristic of easier technology for conjugating polymer and cargo has become the most typical and effective linker to promote particles decomposition and agents rapid release. ${ }^{71}$ Additionally, upregulated enzymes also serve as key stimuli for triggering signal turn-on with the significantly reduction of off-target effect owing to the high specificity of enzyme catalysis. ${ }^{69}$ The cellular triggered reactions are designed in advance in the physical model, in which the macrostructures of the NPs changed by the over-expressed enzymes can result in a rapid release of payloads through transformation or degradation when the NPs reach the cytoplasm. ${ }^{72}$ Among them, hydrolase and redox enzymes are more sensitive which are generally introduced to construct enzyme-triggered responsive NPs. Moreover, some stimulus responsive DDSs are based on the high concentrations of ATP and high level of reactive oxygen species (ROS) in the cytoplasm. ${ }^{73}$ Based on cellular and subcellular-triggers stimuli, $\mathrm{Wu}$ et al prepared a GSH/ Hyals/pH-responsive NPs (NX@Cs-SNX/cHA) composed of chitosan/hyaluronan (HA) for effective SNX2112 (an inhibitor of heat shock protein 90 in breast cancer cells) release within tumor cells with good stability of bloodstream and intracellular environment sensitivity. ${ }^{74,75} \mathrm{HA}$ as the NPs' shell conjugated with chitosan core by disulfide bonds. Negatively charged HA could prolong the circulation of NPs in blood and specifically bind to CD44 that 


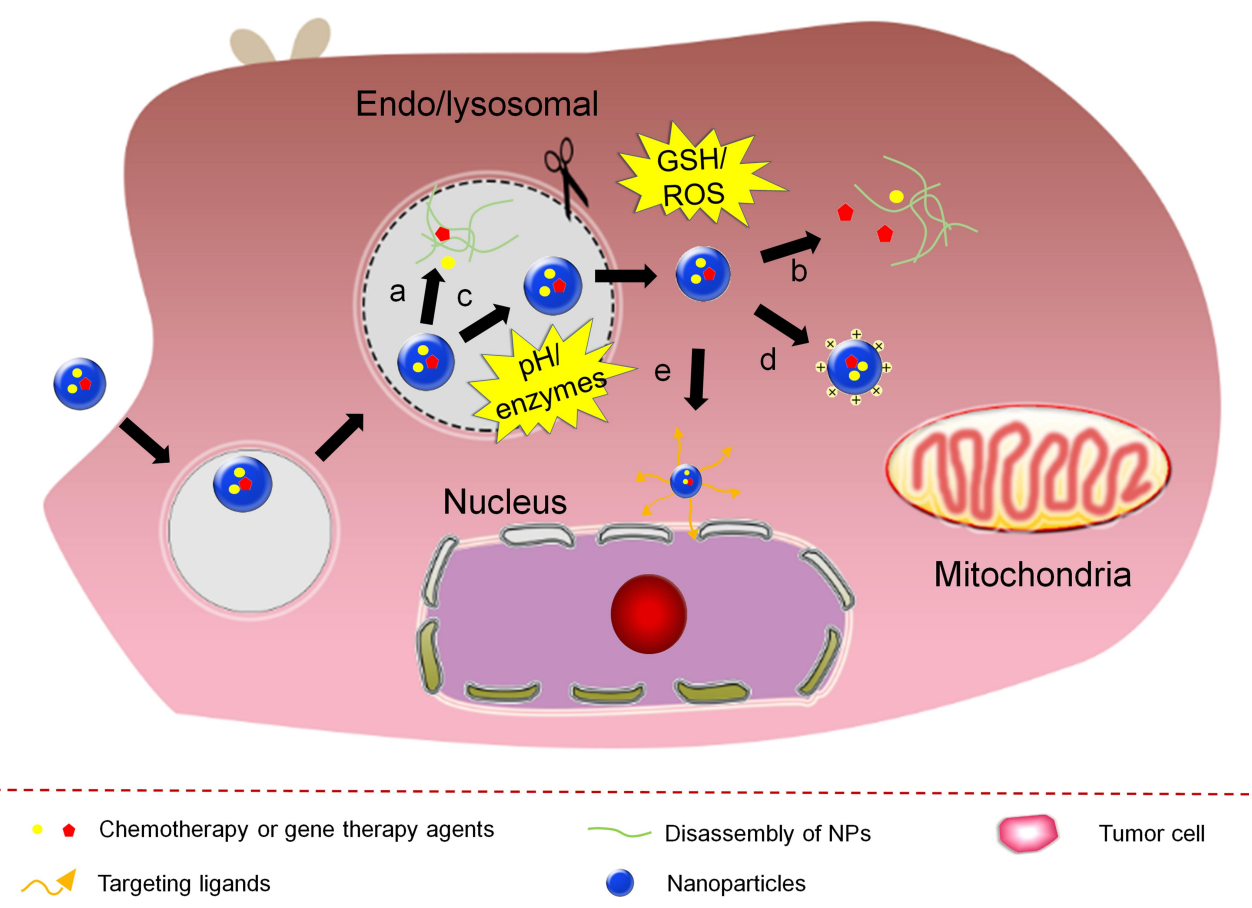

Figure 7 MSR-DDSs responsive to cellular and subcellular triggers for accelerated agents release or cellular substructural targeting. NPs disintegration for agents release (a and b); endosomal escape (c), mitochondrial targeting (d) and nuclear localization (e).

contributed to target breast cancer cells. HA was degraded by hyaluronidase (Hyals) and the disulfide bonds were destroyed by GSH reduction when NX@Cs-SNX/cHA entered into tumor cells, exposing the $\mathrm{pH}$-unstable core which facilitated the release of SNX2112 in the acidic environment ( $\mathrm{pH}$ 5.0) and the escape from endosomes/ lysosomes. In mimicking the tumor intracellular environment, NX@Cs-SNX/cHA released 84\% of payload within $72 \mathrm{~h}$, as opposed to $28 \%$ at $\mathrm{pH} 7.4$ condition. The dualcellular triggers responsive NPs can realize the rapid delivery of agents within cancer cells. Additional MSR-DDSs triggered by cellular and subcellular-stimuli for delivering antitumor agents are listed in Table 1.

\section{MSR-DDSs for Tumor Theranostics}

Theranostics of renown is well known as simultaneous diagnosis/imaging and treatment, which emerged out of clinical necessities to monitor the real-time state of smart NPs in vivo under different stimuli and avoid unexpected situations that do not operate according to the designs, such as off-target effect, non-response. MSR-DDSs for theranostics can be applied not only for the precise treatment of tumors but also provide a patient-centered, whole-course solution. Multimodal imaging can integrate structure/function information to reduce the influence of complex biological environments, high background signals, and probe concentration (as well as avoid the interference of multiple signals generated by itself) to ensure a more accurate diagnosis. ${ }^{96}$ Light and magnetic fields are important for MRI and photoacoustic imaging to guide imaging and diagnosis in theranostics, as well as for triggering release of agents and converting heat to kill tumor cells directly. Theranostics, multimodal imaging, and realtime monitoring of agents in vivo can be achieved by removing shielding (quenching agents) or disintegrating NPs to expose materials by making exogenous triggers emit light, or release ligands to weaken the signal transmission of the NPs themselves.

Recent studies have revealed that mild hyperthermia ($39-42^{\circ} \mathrm{C}$ ) can be used in MSR-DDSs for theranostics by boosting the permeability of cell membranes and blood vessels to improve tumor therapy and cellular uptake. ${ }^{97}$ Melanin has strong absorption and thermal conversion in the NIR region, and it could be used for PAI and PTT. ${ }^{98}$ Feng and coworkers designed $\mathrm{pH} / \mathrm{NIR}$-responsive melanin-coated magnetic NPs (MMNs) to deliver an inhibitor of wingless type (Wnt)/ $\beta$-catenin signaling, obatoclax (OBX), by $\pi-\pi$ stacking and hydrophobic interactions which afforded magnetic resonance (MR)/photoacoustic dual-mode imaging for further accurate tumor diagnosis and guided chemotherapy 
enhanced by mild hyperthermia. ${ }^{98,99}$ Interestingly, blockade of Wnt signaling could control mammary tumor virus-Wnt1 transgenic tumors in mice. Changes in the NIR region and $\mathrm{pH}$ could work together to accelerate OBX release. Mild hyperthermia was also conducive to OBXMMN accumulation in tumors and cell internalization, OBXMMNs could suppress proliferation of breast-cancer cells upon chemical-photothermal therapy. The photoacoustic intensity increased with an increasing concentration of OBXMMNs, but T2-weighted MR signals were negatively correlated with the OBX-MMN concentration. This dual-mode imaging platform can provide more accurate imaging and diagnosis than that using a single-mode platform. NIR light has deep penetration in biological tissues due to weak absorption, which is conducive to the initiation of light-activated chemotherapy. ${ }^{100}$ The up-conversion method of transforming NIR light into photoactivated chemotherapy at the lesion site has attracted much attention. ${ }^{101} \mathrm{Xu}$ et al prepared UCNPs with reversible charge and shrinking size to better promote cell internalization. ${ }^{102}$ The hydrolysis of PEG-PAHDMMA layer emerged UCNs loaded Pt(IV) prodrug by charge reversed in TME. Positive UCNPs-Pt(IV) had high affinity for negatively charged cell membranes, which resulted in effective internalization. Simultaneously, lanthanide-doped UCNPs effectively absorbed NIR light, then emitted UV light and all ranges of visible light. UV light and GSH within cancer cells transformed the Pt(IV) prodrug to $\mathrm{Pt}(\mathrm{II})$ with high cytotoxicity. In addition, application of $\mathrm{Yb}^{3+}$-doped materials with strong $\mathrm{X}$-ray attenuation and high atomic mass can be used as contrast agents for computed tomography (CT). ${ }^{103}$ UCNPs enable integration of photoactivated chemotherapy and CT/up-conversion luminescence dual-mode imaging. Dong et al used a simple chelating method to label ${ }^{64} \mathrm{Cu}$ on nano-surfaces to monitor their accumulation, distribution, and response in real time in tumor-bearing mice by single-photon emission computed tomography (Figures 8A and B). ${ }^{104,105}$ The nano-platform comprised $\mathrm{MoS}_{2}$ nano-thermally permeable materials modified with HA targeting MCF-7-ADR. $\mathrm{MoS}_{2}$ has strong photothermal-conversion ability in the NIR region and can promote DOX release under laser irradiation at $808 \mathrm{~nm}, \mathrm{HA}$ can also be disintegrated by HAase in the TME to accelerate DOX release. ${ }^{106}$ Expression of multidrug resistance-related P-glycoprotein (P-gp) was downregulated in response to
A

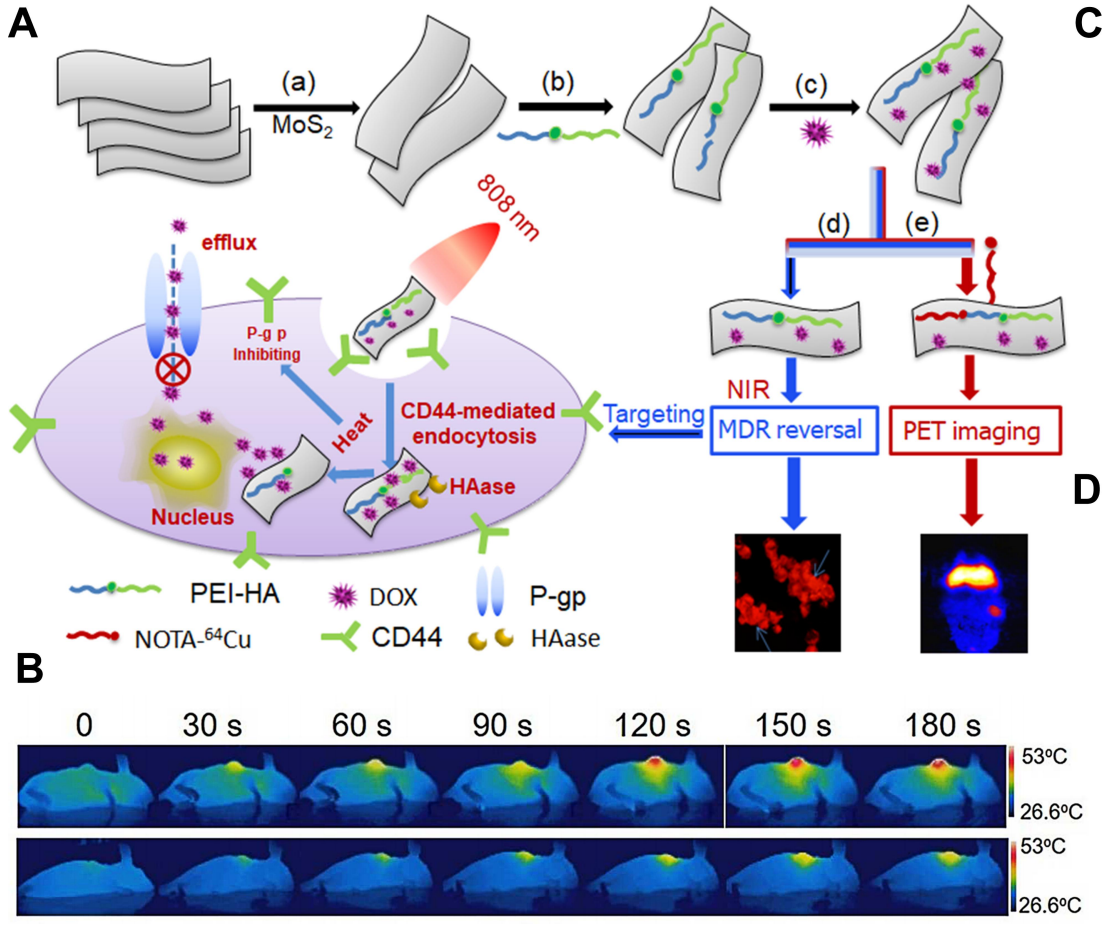

Figure 8 The design strategy of $\mathrm{MoS}_{2}$-PEI-HA nanosheets. (A) Synthesis of MoS 2 -PEI-HA nanosheets (a-c); (d) MoS 2 -PEI-HA NPs target CD44 cells and induce MDR reversal; (e) ${ }^{64} \mathrm{Cu}$ mediated PET imaging. (B) Infrared thermal imaging of tumor-bearing mice. (C) Expression level of P-gP protein. (D) Average tumors weight of each

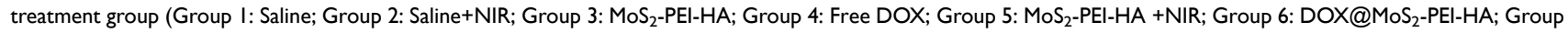
7: DOX@MoS 2 -PEI-HA +NIR). (*p < 0.05).

Notes: Reprinted from Dong X, Yin W, Zhang X, et al. Intelligent MoS(2) Nanotheranostic for Targeted and Enzyme-/pH-/NIR-Responsive Drug Delivery To Overcome Cancer Chemotherapy Resistance Guided by PET Imaging. ACS Applied Materials \& Interfaces. 2018;10(4):427I-4284. Copyright (2018), with permission from ACS. ${ }^{105}$ 
HAase/NIR (Figure 8C). DOX@MoS2-PEI-HA+NIR exhibited an approximately $96 \%$ of tumor inhibition ratio, comparing with $76.0 \%$ of tumor inhibition ratio of DOX@MoS2-PEI-HA (Figure 8D). This action resulted in a significant increase in intracellular drug accumulation and reversal of drug resistance with excellent anti-tumor activity.

Zhao et al designed and prepared a theranostic nanoprobe for precision diagnostics. It could be delivered to the tumor site as a drug carrier and achieved a combination of rapid detection and direct treatment of tumors. ${ }^{107}$ The reversible $\mathrm{pH}$-responsive NIR absorption and fluorescence of asymmetric cyanine have enabled its use as a tumorspecific imaging probe and auxiliary photothermal agent. GNRs are characterized by high photothermal conversion efficiency and strong NIR absorption. Photothermal therapeutic agents and ultra-efficient quenchers were conjugated through MMP-specific lysates peptides $\left(\mathrm{H}_{2}\right.$ N-GPLGVRGC-SH; PLGVR is the cleavable site) to construct a theranostic nanometer probe. The latter was synergistically and reversibly activated by $\mathrm{MMPs} / \mathrm{pH}$ to produce a synergistic photothermal effect between GNRs and asymmetric cyano groups. Notably, one of the most important nutrient transporters, D-glucose transporter (GLUT), shows high expression in the TME. GLUT could bind to the glycosyl group to confer satisfactory tumor targeting and biodistribution of this nanoprobe. The prepared theranostic nanoprobe possessed promising tumor-targeting performance with high specificity and extremely low background but also had a robust photothermal effect with very little systemic toxicity. MSRDDSs have broad application prospects in theranostics. $\mathrm{Cu}_{2} \mathrm{~S}$-based MSR-DDSs for theranostics with adjustable sizes and shapes can burst-release a drug in response to multiple stimuli and exhibit superiority in photoacoustic, optical, and X-ray imaging systems. They have good biocompatibility and high absorbance for the imaging and multimodal treatment of brain-tumor cells. ${ }^{108}$

Monitoring and tracking the details of intracellular drug release are also vital for improvement of MSRDDSs. Cao and coworkers reported real-time monitoring of controlled release of intracellular DOX and 6-mercaptopurine (6MP) by Janus NPs based on fluorescence resonance energy transfer (FRET) and surface-enhanced Raman scattering (SERS). ${ }^{109}$ FRET helps in monitoring the change in distance of donor-acceptor separation at $<10$ $\mathrm{nm}$ as a recognized energy-transfer process between two fluorophores. ${ }^{110}$ SERS can boost the Raman signals of molecules by $6-14$ orders of magnitude if it is adsorbed on gold or silver nanostructures. ${ }^{111}$ DOX (FRET acceptor) was conjugated onto 7-hydroxycoumarin-3-carboxylate (CMR; FRET donor)-functionalized NPs through a $\mathrm{pH}-$ responsive linker hydrazone bond. 6MP was connected to the gold surface via gold-mercaptan interactions. Then, the surface of NPs was decorated further to conjugate 6MP-coated gold. When Janus NPs entered tumor cells, the breakage of hydrazone bonds and substitution of GSH resulted in effective release of DOX and 6MP, respectively. The change of the fluorescence signal of CMR and SERS signal of 6MP based on the distance between two molecules/substances can be used to monitor the release of two drugs in living cells in real time. MSRDDSs can accumulate at the disease site, which has shown great promise in accurate imaging for theranostics. Additional MSR-DDSs for theranostics are listed in Table 2.

\section{MSR-DDSs for Tumor Immunotherapy}

Tumor immunotherapy is rapidly becoming a promising cancer treatment strategy through anti-tumor immune response (ACIR) that reflects the recognition, proliferation, and activation of immune cells, effector $\mathrm{T}$ cells' migration and infiltration to the tumor tissue, ultimately targeting, fighting and elimination of cancer cells without destroying healthy cells. ${ }^{124}$ Despite the human immune system is strong, the TME will still evade the immune system in many ways through reprogramming, for example, activating immune checkpoint proteins, polarizing macrophages for tumorigenic macrophages, neutralizing cytotoxic $\mathrm{CD}^{+} \mathrm{T}$ cells, highly expressing Indoleamine 2.3-Dioxygenase (IDO), weakening $\mathrm{T}$ cell activity, etc. ${ }^{125,126}$ Moreover, highly heterogeneous and hypoxic TME also inhibits antigen uptake and presentation, promoting the release of immunosuppressive cytokines from tumors and stromal cells, which restricts the tumor infiltration of $\mathrm{T}$ cells to result in the inhibition of ACIR. ${ }^{125}$ So the strategies of immunotherapy can mainly be divided into two categories: (1) Inhibition of tumor immune escape; (2) Promoting ACIR by improving tumor immunogenicity or providing immune-stimuli agents to directly activate the activity of the immune system, and so on. To achieve targeted delivery and co-delivery with less adverse autoreactive immune responses, immunotherapeutic agents can be delivered on the platform of MSR-DDSs. 
Table 2 Additional MSR-DDSs for Theranostics

\begin{tabular}{|c|c|c|c|c|}
\hline Stimuli & $\begin{array}{l}\text { Materials That } \\
\text { Produce Signal }\end{array}$ & Monitoring Methods & Means of Therapy & References \\
\hline $\mathrm{NIR/GSH}$ & GO & PL & Gene therapy & {$[112]$} \\
\hline Light/pH & ICG & NIRF imaging & Chemotherapy and PTT & [113] \\
\hline Magnetic/NIR & 10 & $\mathrm{MRI}$ and PAI & Chemotherapy and PTT & {$[114]$} \\
\hline Light/GSH & $\mathrm{MgPc}$ & $\mathrm{FL}$ & Chemotherapy and PTT, PDT & [115] \\
\hline $\mathrm{NIR} / \mathrm{pH}$ & Gold nanorods and ICG & $\begin{array}{l}\text { Two-photon luminescence, FL, photoacoustic } \\
\text { photothermal imaging }\end{array}$ & Chemotherapy and PTT, PDT & [116] \\
\hline $\mathrm{NIR} / \mathrm{pH}$ & DPCN & Multiple imaging & Chemotherapy and PTT & {$[117]$} \\
\hline $\mathrm{pH} / \mathrm{GSH} /$ light & PDA coated $\mathrm{Fe}_{3} \mathrm{O}_{4} \mathrm{NPs}$ & Magnetic and thermal imaging & Chemotherapy and PTT & {$[118]$} \\
\hline $\mathrm{pH} / \mathrm{GSH} / \mathrm{H}_{2} \mathrm{O}_{2}$ & CDs & $\mathrm{FL}$ & PDT, PTT, CDT & [119] \\
\hline $\mathrm{pH} /$ thermal & $\mathrm{Fe}_{3} \mathrm{O}_{4} @ \mathrm{Au} \mathrm{NPs}$ & $M R I$ and $\mathrm{PI}$ & Chemotherapy and PTT, PDT & {$[120]$} \\
\hline $\begin{array}{l}\mathrm{pH} / \mathrm{ROS} / \mathrm{GSH} / \mathrm{HAase} / \\
\text { hyperthermia }\end{array}$ & NIR770 & NIRI, PTI and PAI & Chemotherapy and PTT, PDT & {$[12 \mid]$} \\
\hline $\mathrm{pH} / \mathrm{NIR}$ & $\begin{array}{l}\mathrm{Gd}^{3+} \text {-doped } \mathrm{NaxWO}_{3} \\
\text { nanorods }\end{array}$ & MRI & Chemotherapy and PTT, RT & {$[122]$} \\
\hline GSH/NIR & Mn-Doped NPs & MRI & Chemotherapy and PTT & [123] \\
\hline
\end{tabular}

Abbreviations: GO, graphene oxide; PL, photoluminescence; NIRF, near-infrared; FL, fluorescence imaging; 10-HCPT, I0-hydroxycamptothecin; IO, superparamagnetic iron oxide; PET, photon emission computed tomography; MgPc, magnesium phthalocyanine; DPCN, dendritic platinum-copper alloy nanoparticles; CDs, carbon dots; CDT, chemodynamic therapy; Gd, gadolinium; RT, radiotherapy; UTMD, ultrasound-targeted microbubble destruction.

The human body produces an immune response after being stimulated and activated by a foreign substance. Nevertheless, the immune system cannot be activated excessively owing to immune-checkpoint proteins (eg, programmed cell death-1 (PD-1), CTLA-4, LAG3), which can produce immunosuppressive cytokines. ${ }^{125}$ Tumors can inhibit the activity of the immune system with immune escape by tumor cellular ligands binding to the PD-1 protein, which is expressed in $\mathrm{T}$ cells and $\mathrm{B}$ cells. ${ }^{126}$ Besides immune-checkpoint proteins, transforming the essential amino acid 1-tryptophan to 1-kynurenine through highly expressed IDO in solid tumors can also inhibit the proliferation and activity of $\mathrm{T}$ cells, so as to promote immune escape of tumor cells. ${ }^{127}$ Cheng et al reported on sequenceresponsive peptide-based NPs for dual-targeted tumor immunotherapy, which contributed to superior melanoma inhibition with an enhanced level of cytotoxic $\mathrm{T}$ cells by simultaneously blocking immune checkpoints and suppressing IDO (Figure 9A). ${ }^{128}$ Amphiphilic DEAP-DPPA-1 consisting of 3-diethylaminopropyl isothiocyanate (DEAP), MMP-2 cleavable linker and an antagonist of PD-1

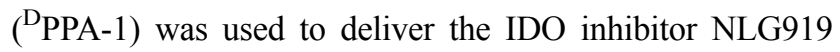

as a co-assembly carrier. The functional group DEAP of DEAP- ${ }^{\mathrm{D} P P A-1}$ was protonated by an acidic TME. The hydrophobicity of NPs was reduced while the structure swelled. Further dissociation of DEAP- ${ }^{\mathrm{D} P P A}-1$ by the presence of MMP-2 caused the nanostructure to collapse com-

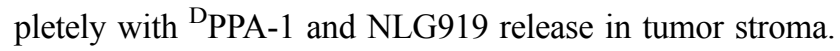
NLG919@DEAP-DPA-1 had superior antitumor activity and could promote more $\mathrm{CD}^{+} \mathrm{T}$ cells differentiation (Figure 9B and C). The hydrophobic fluorescing agent TRITC and "black hole" quencher were also assembled into NPs to further evaluate the precision of tumorresponsive potential. ${ }^{129}$ Interestingly, the fluorescence of TRITC could be restrained by vicinal BHQ-1 by intermolecular energy transfer unless the structure of NPs was destroyed to release fluorescent molecules to result in the conversion of the nanoprobe from a quenched state to an active fluorescent state. Those findings showed that the NPs were only disassembled at the tumor site according to ex vivo imaging. Those results demonstrated that encapsulation of NLG919 by DEAP- ${ }^{\text {DPPA-1 }}$ was an effective tumorimmunotherapy platform for tumor localization and survival improvement by precise control of agents. 


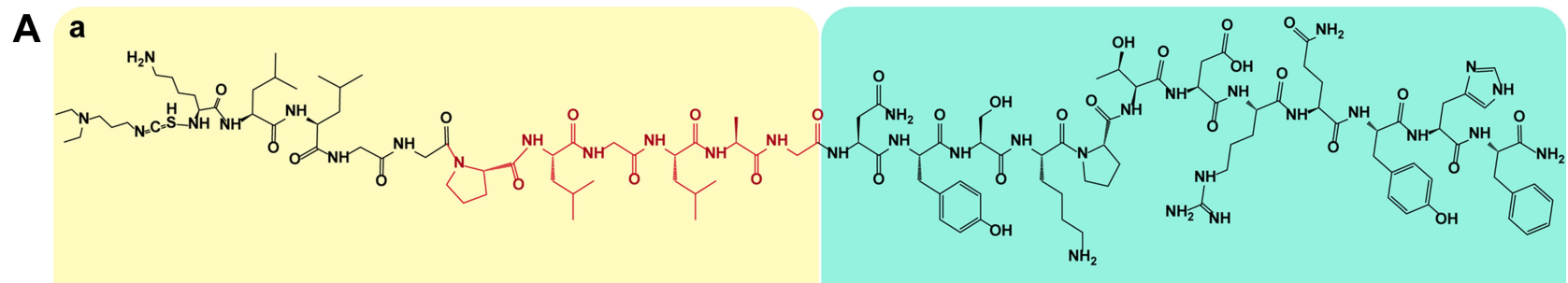

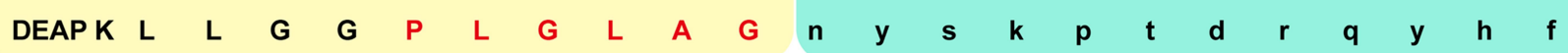

b

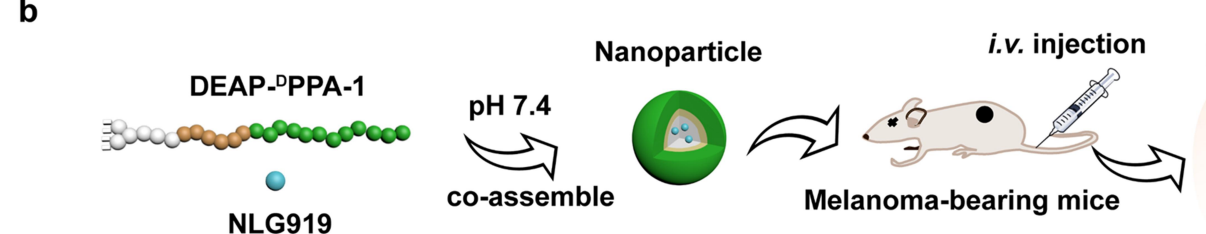

Stable nanostructure in physiological environment
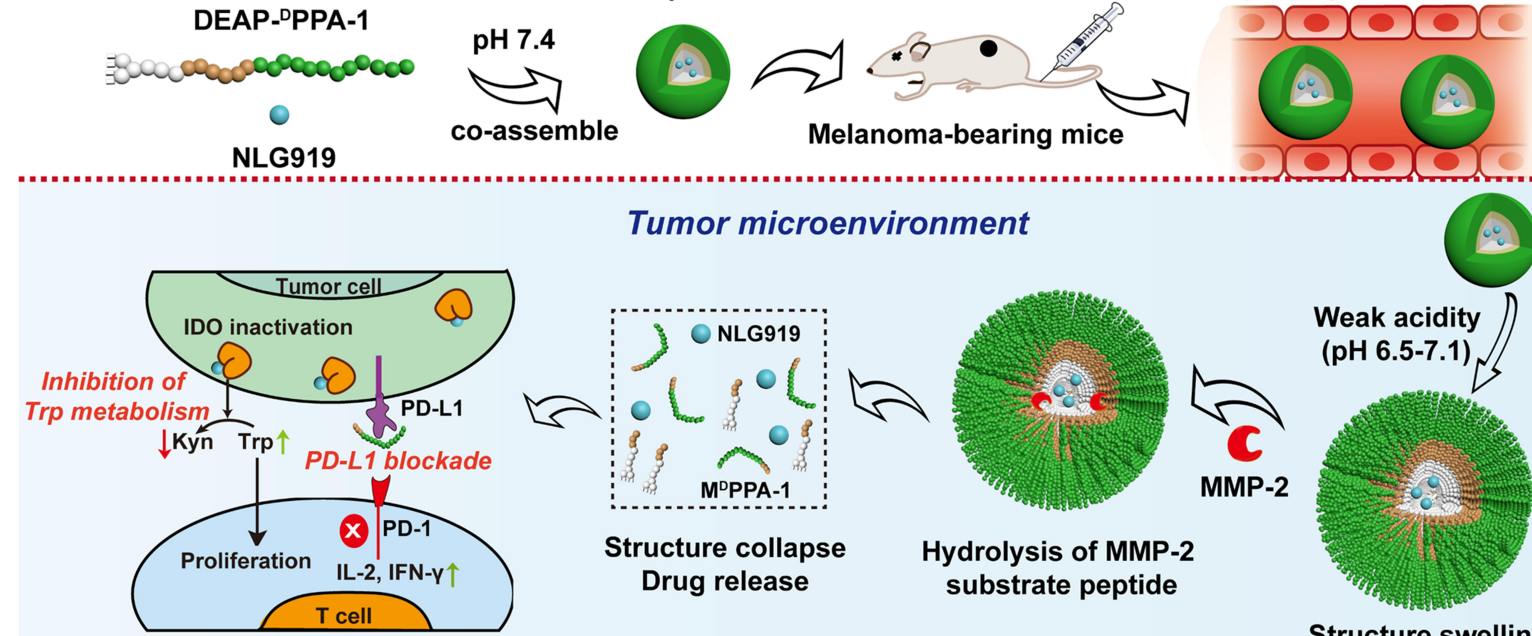

Tumor microenvironment

Stimulation of $\mathrm{T}$ cell proliferation and activation
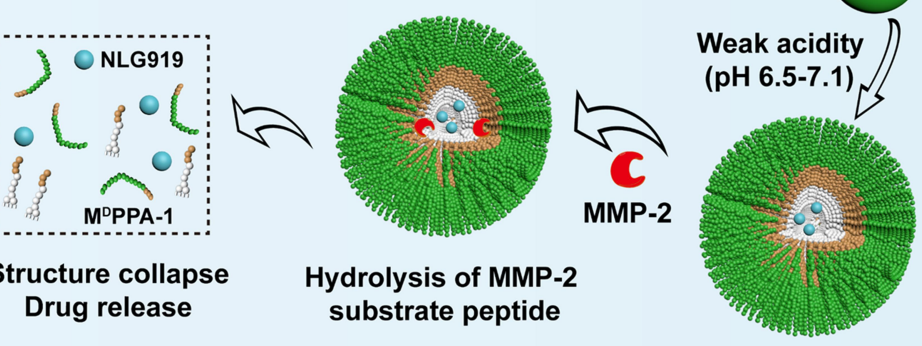

Structure swelling

B

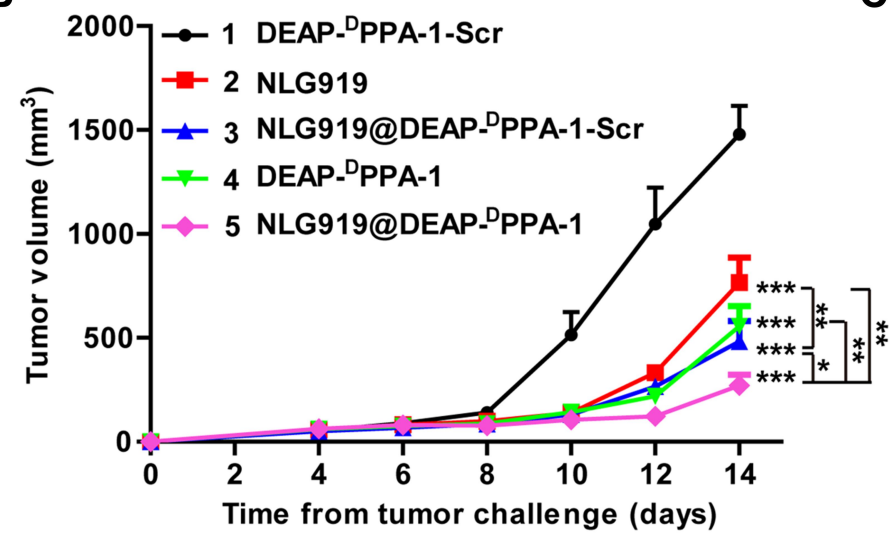

C

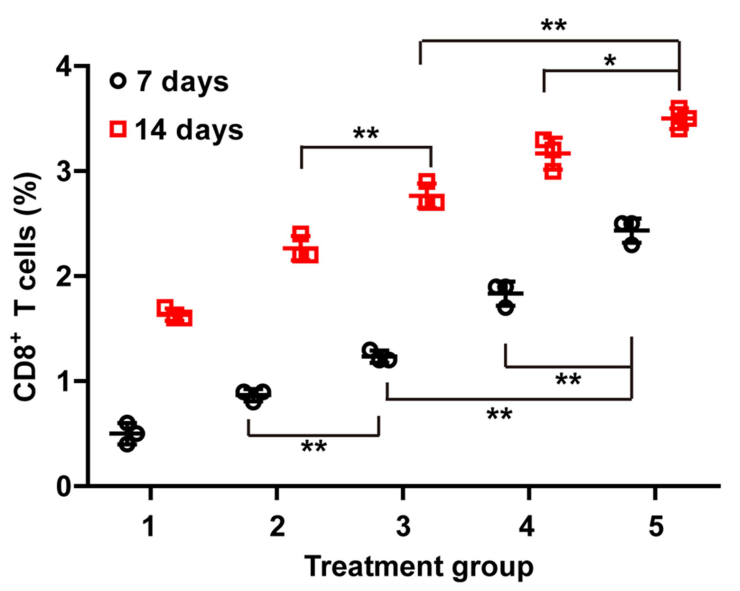

Figure 9 The design strategy of NLG919@DEAP-DPPA-I. (A) The responding model and antitumor mechanism of NLG919@DEAP-DPPA-I; (B) tumor volume growth curves of mice after different treatments; $(\mathbf{C})$ the ratio of $C D 8^{+} \mathrm{T}$ cells after treatment. $\left({ }^{*} \mathrm{p}<0.05, * * \mathrm{p}<0.0 \mathrm{I}, *^{* * *} \mathrm{p}<0.00 \mathrm{I}\right)$.

Notes: Reprinted from Cheng K, Ding Y, Zhao Y, et al. Sequentially Responsive Therapeutic Peptide Assembling Nanoparticles for Dual-Targeted Cancer Immunotherapy. Nano Letters. 2018;18(5):3250-3258. Copyright (2018), with permission from ACS. ${ }^{128}$

An immunogenic "hot" TME infiltrated with specific $\mathrm{T}$ cells can produce ACIR. ${ }^{130}$ However, many patients have immunogenic "cold" tumors lacking significant infiltration of $\mathrm{T}$ cells. The TME predominantly comprises high-density immunosuppressive cells. Therefore, reprogramming of cold tumors into immunogenic and proinflammatory states has become a major underlying factor to improve immunotherapy. In recent years, several anticancer drugs (eg, anthracycline, oxaliplatin) have been reported to induce antigen release during immunogenetic cell death (ICD) against cold tumors, which can be used for ACIR, as well as a combination of chemotherapy and 
immunotherapy. ${ }^{131}$ Zheng et al synthesized folatemodified monodispersed silica NPs with $\mathrm{pH}$ - and GSHresponsiveness to target DOX release for inducing ICD and maximum immunity. ${ }^{132}$ The intracellular environment triggered release of DOX to induce apoptosis, Box-1 release and subsequently release antigens with high mobility from dead cells for activating antigen-presenting cells, generating ACIR, and further eliminating tumor cells. Exogenous photodynamic therapy (PDT) can also be introduced to activate ICD. Hou et al induced ICD to promote the infiltration of tumor cytotoxic $\mathrm{T}$ lymphocytes by PDT based on higher-order Boolean logic prodrug NPs (BLPNs), which could activate pheophorbide a (photosensitizer) prodrug and release NLG919 in response to intracellular redox to activate the immune system. ${ }^{133}$ The strategies of triggering BLPNs can be classified mainly into three parts: (i) MMP-2/9 cleavage of the PEG corona of BLPNs, which promotes the accumulation and retention of BLPNs in tumor tissue based on enhanced targeting; (ii) degradable disulfide bonds are triggered by intracellular GSH to release NLG919; (iii) acid sensitive 2-(diisopropylamino)ethyl methacrylate can be protonated to dissociate NPs and active pheophorbide a for PDT. PDT induced tumor cell apoptosis with ICD generation to increase tumor immunogenicity, and the introduction of NLG919 inhibited the activity of IDO to prevent the immune escape of tumor. $^{134}$ The co-delivery of PPa and NLG919 can be coordinately used to modulate the tumor immune microenvironment for both ACIR and anti-immune escape. The results of monitoring the secretion levels of IFN- $\gamma$, TNF- $\alpha$, and IL-6, proved that the synergistic effect of BLPNmediated NLG919 transmission and PDT can cause obvious immunoinflammatory response to induce tumor cell apoptosis. In immunotherapy, it is crucial to elicit the tumor immunogenicity to activate the ACIR, it is also a key factor that the activation of antigen-presenting cells (such as DC) depending on triggering the pattern recognition receptors (such as TLR). Mature DC cells can migrate to the lymph nodes and activate $\mathrm{T}$ cells with over-expressing surface molecules (such as CD80/CD86) to produce anti-tumor immunity. ${ }^{135}$ R848 is a TLR7/8 agonist that can promote the maturation and enhance the function of DC through myeloid differentiation factor (MyD88) pathway. Some studies have shown that R848 receptor agonist can not only enhance the migration of DC but also improve the infiltration level of $\mathrm{CD}^{+}$and $\mathrm{CD}^{+}$ T cells in tumor tissues. ${ }^{136}$ A multi-functional DDS with double $\mathrm{pH}$ responses was designed by Liu's group to treat breast cancer based on combining chemotherapy and immunotherapy. ${ }^{137}$ The NPs were composed of polymeric prodrug HA-DOX shell and poly(L-histidine) (PHIS) formed nanocores with R848 loaded by hydrazone bonds. In the weakly acidic TME, the hydrophobic/hydrophilic transformation of PHIS triggered the disintegration of NPs, which in turn triggered the release of R848 and exerted its anti-tumor immune activity. The internalization of HA-DOX was induced through CD44-mediated DOX endocytosis, then the hydrazone bonds were broken within intracellular acid environment resulting in a rapid release of DOX to further kill tumor cells. Finally, extensive verifications have proved that the HA-DOX/PHIS/R848 NPs exerted their anti-tumor immune activity by promoting the activation of DC cells and increasing the infiltration of $\mathrm{T}$ cells in TME.

MSR-DDSs provide a novel proposition for accurate tumor immunotherapy, MSR-DDSs strategy also pave the way for combination inhibition of different immune resistance mechanisms or chemotherapy.

\section{Conclusions and Outlook}

Recent advances in nanotechnology and novel stimuliresponsive polymeric materials have allowed engineering of smart NPs for the diagnosis and treatment of cancer. MSR-DDSs integrate the versatility of different strategies into a multifunctional nano-system. The latter can achieve modular and spatiotemporal control. NPs can undergo charge reversal, size shrinkage, PEG detachment, reemergence of ligand targeting, and reactive drug release. Likewise, they provide a good platform for maximizing the tumoricidal effects of chemotherapy, gene interference, and immune regulation, as well as realize the application of combinations. Moreover, MSR-DDSs have been designed for theranostics and multimodal imaging, which address many of the difficulties associated with a single imaging model (eg, poor resolution or sensitivity). ${ }^{12,138}$

So far, about fifty nano-preparations have been approved for clinic application, such as Doxil (Liposomal/DOX), Marqibo (Liposomal/Vincristine), Abraxane (Nanoparticulate albumin/ PTX), Lipusu (Liposomal/PTX), and so on. ${ }^{139-141}$ They are either actively or passively targeted to improve the effect of cancer treatment or reduce side effects. Although many achievements have been made in smart DDSs so far, what reported mostly are proof-of-concept studies as the relative age of nanotechnologies. Visudyne (UV light-responsive) was the only one approved by FDA among smart DDSs, Opaxio (enzymeresponsive), NanoTherm (magnetic-responsive), ThermoDox 
(temperature-responsive) and others are all in clinical trials stage. ${ }^{142-145}$ Hence, some issues still need to be addressed from the laboratory to clinical applications for the follow-on innovation, scientific and technical challenges are summarized as follows: (1) Individual differences of patients, different stages of disease development as well as the cellular heterogeneity of tumor cells may generate different drug delivery efficiency; ${ }^{146}$ (2) The expression and distribution of stimuli in TME are highly heterogeneous and dynamically variable, which may lead to unanticipated stimuli response; (3) Low $\mathrm{pH}$, high reductant concentration or up-regulation of enzymes in normal cells or tissues may induce off-target effects; (4) Whether stimuli-responsive polymeric materials are safe in vivo, the breakdown and longevity of nanocarriers need to be considered carefully to avoid long-term medical downsides; (5) Due to the differences in genetic background between mice and humans, the tumor models often cannot recapitulate the clinical situation of patients, thereby the transformation from animal models to clinical treatment is complex. ${ }^{147}$ Although MSR-DDSs can greatly reduce the impact of individual differences and tumor heterogeneity with less off-target tissues compared with single-stimulus responsive NPs, the sophisticated designs limit their application as industrial production and quality control difficult on a large scale. It is urgent to design rational MSR-DDSs with simple preparation craft. Further specific stimuli and novel stimuli combinations should be screened for improving the accuracy of agents release, reducing the impacts of individual differences and different treatment stages to adapt to complex clinical applications. Reviewing the emerging trends of MSR-DDSs can accelerate innovation of nanomaterials for medical applications, and identify the trends or class-based toxicity profiles of nanomaterials. The next 20-30 years are looking bright for cancer patients.

\section{Acknowledgments}

This work was financially supported by the Xinglin Scholar Engineering of Changchun University of Chinese Medicine; the Jilin Provincial Science and Technology Department Project, [Grant No. 20190103073JH].

\section{Disclosure}

The authors report no conflicts of interest in this work.

\section{References}

1. Bray F, Ferlay J, Soerjomataram I, Siegel RL, Torre LA, Jemal A. Global cancer statistics 2018: GLOBOCAN estimates of incidence and mortality worldwide for 36 cancers in 185 countries. CA Cancer J Clin. 2018;68(6):394-424. doi:10.3322/caac.21492
2. Iyer AK, Singh A, Ganta S, Amiji MM. Role of integrated cancer nanomedicine in overcoming drug resistance. Adv Drug Deliv Rev. 2013;65(13-14):1784-1802. doi:10.1016/j.addr.201 3.07.012

3. Yang Z, Shi J, Xie J, et al. Large-scale generation of functional mRNA-encapsulating exosomes via cellular nanoporation. Nat Biomed Eng. 2020;4(1):69-83. doi:10.1038/s41551-019-0485-1

4. Chanmee T, Ontong P, Konno K, Itano N. Tumor-associated macrophages as major players in the tumor microenvironment. Cancers. 2014;6(3):1670-1690. doi:10.3390/cancers6031670

5. Wu J, Chen J, Feng Y, Tian H, Chen X. Tumor microenvironment as the "regulator" and "target" for gene therapy. J Gene Med. 2019;21(7):e3088. doi:10.1002/jgm.3088

6. Karagiannis GS, Poutahidis T, Erdman SE, Kirsch R, Riddell RH, Diamandis EP. Cancer-associated fibroblasts drive the progression of metastasis through both paracrine and mechanical pressure on cancer tissue. Mol Cancer Res. 2012;10(11):1403-1418. doi:10.1158/1541-7786.Mcr-12-0307

7. Ivey JW, Bonakdar M, Kanitkar A, Davalos RV, Verbridge SS Improving cancer therapies by targeting the physical and chemical hallmarks of the tumor microenvironment. Cancer Lett. 2016;380(1):330-339. doi:10.1016/j.canlet.2015.12.019

8. Cabral H, Matsumoto Y, Mizuno K, et al. Accumulation of sub-100 $\mathrm{nm}$ polymeric micelles in poorly permeable tumours depends on size. Nat Nanotechnol. 2011;6(12):815-823. doi:10.1038/nnano.2011.166

9. Rosenblum D, Joshi N, Tao W, Karp JM, Peer D. Progress and challenges towards targeted delivery of cancer therapeutics. Nat Commun. 2018;9(1):1410. doi:10.1038/s41467-018-03705-y

10. Li L, Wang J, Kong H, Zeng Y, Liu G. Functional biomimetic nanoparticles for drug delivery and theranostic applications in cancer treatment. Sci Technol Adv Mater. 2018;19(1):771-790. doi:10.1080/14686996.2018.1528850

11. Jain RK, Stylianopoulos T. Delivering nanomedicine to solid tumors. Nat Rev Clin Oncol. 2010;7(11):653-664. doi:10.1038/ nrclinonc.2010.139

12. El-Sawy HS, Al-Abd AM, Ahmed TA, El-Say KM, Torchilin VP. Stimuli-responsive nano-architecture drug-delivery systems to solid tumor micromilieu: past, present, and future perspectives. ACS Nano. 2018;12(11):10636-10664. doi:10.1021/acsnano.8b0 6104

13. Li F, Yang H, Bie N, et al. Zwitterionic temperature/redox-sensitive nanogels for near-infrared light-triggered synergistic thermo-chemotherapy. ACS Appl Mater Interfaces. 2017;9 (28):23564-23573. doi:10.1021/acsami.7b08047

14. Vo KT, Matthay KK, DuBois SG. Targeted antiangiogenic agents in combination with cytotoxic chemotherapy in preclinical and clinical studies in sarcoma. Clin Sarcoma Res. 2016;6:9. doi:10.1186/s13569-016-0049-z

15. Jhaveri A, Deshpande P, Torchilin V. Stimuli-sensitive nanopreparations for combination cancer therapy. $J$ Control Release. 2014;190:352-370. doi:10.1016/j.jconrel.2014.05.002

16. Mura S, Nicolas J, Couvreur P. Stimuli-responsive nanocarriers for drug delivery. Nat Mater. 2013;12(11):991-1003. doi:10.1038/ nmat3776

17. Chen B, Dai W, He B, et al. Current multistage drug delivery systems based on the tumor microenvironment. Theranostics. 2017;7(3):538-558. doi:10.7150/thno.16684

18. Raza A, Rasheed T, Nabeel F, Hayat U, Bilal M, Iqbal HMN. Endogenous and exogenous stimuli-responsive drug delivery systems for programmed site-specific release. Molecules (Basel, Switzerland). 2019;24(6):1117. doi:10.3390/molecules24061117

19. Wang M, Liang Y, Zhang Z, et al. Ag@Fe(3)O(4)@C nanoparticles for multi-modal imaging-guided chemo-photothermal synergistic targeting for cancer therapy. Anal Chim Acta. 2019; 1086:122-132. doi:10.1016/j.aca.2019.08.035 
20. Reguera J, Jiménez de Aberasturi D, Henriksen-Lacey M, et al. Janus plasmonic-magnetic gold-iron oxide nanoparticles as contrast agents for multimodal imaging. Nanoscale. 2017;9 (27):9467-9480. doi:10.1039/c7nr01406f

21. Zhou S, Ding C, Wang C, Fu J. UV-light cross-linked and $\mathrm{pH}$ de-cross-linked coumarin-decorated cationic copolymer grafted mesoporous silica nanoparticles for drug and gene co-delivery in vitro. Mater Sci Eng C Mater Biol Appl. 2020;108:110469. doi:10.1016/j.msec.2019.110469

22. Lei Q, Wang SB, Hu JJ, et al. Stimuli-responsive "Cluster bomb" for programmed tumor therapy. ACS Nano. 2017;11(7): 7201-7214. doi:10.1021/acsnano.7b03088

23. Cheng L, Liu J, Gu X, et al. PEGylated WS(2) nanosheets as a multifunctional theranostic agent for in vivo dual-modal CT/ photoacoustic imaging guided photothermal therapy. Adv Mater. 2014;26(12):1886-1893. doi:10.1002/adma.201304497

24. Sun X, Dong S, Li X, et al. Delivery of siRNA using folate receptor-targeted $\mathrm{pH}$-sensitive polymeric nanoparticles for rheumatoid arthritis therapy. Nanomedicine. 2019;20:102017. doi:10. 1016/j.nano.2019.102017

25. Kakwere H, Materia ME, Curcio A, et al. Dually responsive gold-iron oxide heterodimers: merging stimuli-responsive surface properties with intrinsic inorganic material features. Nanoscale. 2018;10(8):3930-3944. doi:10.1039/c7nr06726g

26. Guardia P, Nitti S, Materia ME, et al. Gold-iron oxide dimers for magnetic hyperthermia: the key role of chloride ions in the synthesis to boost the heating efficiency. $J$ Mater Chem $B$. 2017;5(24):4587-4594. doi:10.1039/c7tb00968b

27. Truong NP, Gu W, Prasadam I, et al. An influenza virus-inspired polymer system for the timed release of siRNA. Nat Commun. 2013;4:1902. doi:10.1038/ncomms2905

28. Dreaden EC, Alkilany AM, Huang X, Murphy CJ, El-Sayed MA. The golden age: gold nanoparticles for biomedicine. Chem Soc Rev. 2012;41(7):2740-2779. doi:10.1039/c1cs15237h

29. Hatakeyama H, Akita H, Kogure K, et al. Development of a novel systemic gene delivery system for cancer therapy with a tumor-specific cleavable PEG-lipid. Gene Ther. 2007;14 (1):68-77. doi:10.1038/sj.gt.3302843

30. Zhou M, Huang H, Wang D, et al. Light-triggered PEGylation/ dePEGylation of the nanocarriers for enhanced tumor penetration. Nano Lett. 2019;19(6):3671-3675. doi:10.1021/acs.nanolett.9b00737

31. Lee Y, Lee S, Lee DY, Yu B, Miao W, Jon S. Multistimuli-responsive bilirubin nanoparticles for anticancer therapy. Angew Chem Int Ed Engl. 2016;55(36):10676-10680. doi:10.1002/anie.201604858

32. Maisels MJ, McDonagh AF. Phototherapy for neonatal jaundice. N Engl J Med. 2008;358(9):920-928. doi:10.1056/NEJMct0708376

33. Sedlak TW, Saleh M, Higginson DS, Paul BD, Juluri KR, Snyder SH. Bilirubin and glutathione have complementary antioxidant and cytoprotective roles. Proc Natl Acad Sci U S A. 2009;106(13):5171-5176. doi:10.1073/pnas.0813132106

34. Zhou X, Chen L, Nie W, et al. Dual-responsive mesoporous silica nanoparticles mediated codelivery of doxorubicin and Bcl-2 SiRNA for targeted treatment of breast cancer. J Phys Chem C. 2016;120(39):22375-22387. doi:10.1021/acs.jpcc.6b06759

35. Feng W, Nie W, He C, et al. Effect of pH-responsive alginate/ chitosan multilayers coating on delivery efficiency, cellular uptake and biodistribution of mesoporous silica nanoparticles based nanocarriers. ACS Appl Mater Interfaces. 2014;6 (11):8447-8460. doi:10.1021/am501337s

36. Ma X, Zhao Y, Ng KW, Zhao Y. Integrated hollow mesoporous silica nanoparticles for target drug/siRNA co-delivery. Chemistry. 2013;19(46):15593-15603. doi:10.1002/chem.201302736

37. Huschka R, Zuloaga J, Knight MW, Brown LV, Nordlander P, Halas NJ. Light-induced release of DNA from gold nanoparticles: nanoshells and nanorods. $J$ Am Chem Soc. 2011;133(31): 12247-12255. doi:10.1021/ja204578e
38. Wang F, Shen Y, Zhang W, et al. Efficient, dual-stimuli responsive cytosolic gene delivery using a RGD modified disulfide-linked polyethylenimine functionalized gold nanorod. J Control Release. 2014;196:37-51. doi:10.1016/j.jconrel.2014.09.026

39. Wang F, Huang Q, Wang Y, Shi L, Shen Y, Guo S. NIR-light and GSH activated cytosolic p65-shRNA delivery for precise treatment of metastatic cancer. J Control Release. 2018;288:126-135. doi:10.1016/j.jconrel.2018.09.002

40. Junttila MR, de Sauvage FJ. Influence of tumour micro-environment heterogeneity on therapeutic response. Nature. 2013;501(7467):346-354. doi:10.1038/nature12626

41. Miao L, Lin CM, Huang L. Stromal barriers and strategies for the delivery of nanomedicine to desmoplastic tumors. $J$ Control Release. 2015;219:192-204. doi:10.1016/j.jconrel.2015.08.017

42. Wang Y, Zhang T, Hou C, et al. Mitochondria-specific anticancer drug delivery based on reduction-activated polyprodrug for enhancing the therapeutic effect of breast cancer chemotherapy. ACS Appl Mater Interfaces. 2019;11(32):29330-29340. doi:10.10 21/acsami.9b10211

43. Ruan S, Cao X, Cun X, et al. Matrix metalloproteinase-sensitive size-shrinkable nanoparticles for deep tumor penetration and $\mathrm{pH}$ triggered doxorubicin release. Biomaterials. 2015;60:100-110. doi:10.1016/j.biomaterials.2015.05.006

44. Yu B, Song N, Hu H, Chen G, Shen Y, Cong H. A degradable triple temperature-, $\mathrm{pH}-$, and redox-responsive drug system for cancer chemotherapy. J Biomed Mater Res A. 2018;106(12):32 03-3210. doi:10.1002/jbm.a.36515

45. Wang Z, Xu Z, Zhu G. A Platinum(IV) anticancer prodrug targeting nucleotide excision repair to overcome cisplatin resistance. Angew Chem Int Ed Engl. 2016;55(50):15564-15568. doi:10.10 02/anie. 201608936

46. Mo R, Sun Q, Xue J, et al. Multistage pH-responsive liposomes for mitochondrial-targeted anticancer drug delivery. Adv Mater. 2012;24(27):3659-3665. doi:10.1002/adma.201201498

47. Callahan J, Kopečkov P, Kopeček J. Intracellular trafficking and subcellular distribution of a large array of HPMA copolymers. Biomacromolecules. 2009;10(7):1704-1714. doi:10.1021/bm80 $1514 \mathrm{x}$

48. Pan L, He Q, Liu J, et al. Nuclear-targeted drug delivery of TAT peptide-conjugated monodisperse mesoporous silica nanoparticles. J Am Chem Soc. 2012;134(13):5722-5725. doi:10. $1021 / \mathrm{ja} 211035 \mathrm{w}$

49. Xie J, Bi Y, Zhang H, et al. Cell-penetrating peptides in diagnosis and treatment of human diseases: from preclinical research to clinical application. Front Pharmacol. 2020;11:697. doi:10.33 89/fphar.2020.00697

50. Lian L, Sun W, Zhong J, et al. Multistage nanovehicle delivery system based on stepwise size reduction and charge reversal for programmed nuclear targeting of systemically administered anticancer drugs. Adv Funct Mater. 2015;25(26):4101-4113. doi:10.10 02/adfm. 201501248

51. Beheshti F, Hassanian SM, Khazaei M, et al. Genetic variation in the DNA repair pathway as a potential determinant of response to platinum-based chemotherapy in breast cancer. $J$ Cell Physiol. 2018;233(4):2752-2758. doi:10.1002/jcp.26091

52. Liu J, Guo X, Luo Z, Zhang J, Li M, Cai K. Hierarchically stimuli-responsive nanovectors for improved tumor penetration and programed tumor therapy. Nanoscale. 2018;10(28):13 737-13750. doi:10.1039/c8nr02971g

53. Liu M, Shen S, Wen D, et al. Hierarchical nanoassemblies-assisted combinational delivery of cytotoxic protein and antibiotic for cancer treatment. Nano Lett. 2018;18 (4):2294-2303. doi:10.1021/acs.nanolett.7b04976

54. Zhao M, Liu Y, Hsieh RS, et al. Clickable protein nanocapsules for targeted delivery of recombinant p53 protein. J Am Chem Soc. 2014;136(43):15319-15325. doi:10.1021/ja508083g 
55. Li HJ, Du JZ, Du XJ, et al. Stimuli-responsive clustered nanoparticles for improved tumor penetration and therapeutic efficacy. Proc Natl Acad Sci U S A. 2016;113(15):4164-4169. doi:10.1073/ pnas. 1522080113

56. Wang M, Alberti K, Sun S, Arellano CL, Xu Q. Combinatorially designed lipid-like nanoparticles for intracellular delivery of cytotoxic protein for cancer therapy. Angew Chem Int Ed Engl. 2014;53(11):2893-2898. doi:10.1002/anie.201311245

57. Lamb R, Ozsvari B, Lisanti CL, et al. Antibiotics that target mitochondria effectively eradicate cancer stem cells, across multiple tumor types: treating cancer like an infectious disease. Oncotarget. 2015;6(7):4569-4584. doi:10.18632/oncotarget.3174

58. Zhang L, Xu L, Zhang F, Vlashi E. Doxycycline inhibits the cancer stem cell phenotype and epithelial-to-mesenchymal transition in breast cancer. Cell Cycle (Georgetown, Tex). 2017;16 (8):737-745. doi:10.1080/15384101.2016.1241929

59. Li Y, Li W, Bao W, et al. Bioinspired peptosomes with programmed stimuli-responses for sequential drug release and high-performance anticancer therapy. Nanoscale. 2017;9 (27):9317-9324. doi:10.1039/c7nr00598a

60. Arpel A, Sawma P, Spenlé C, et al. Transmembrane domain targeting peptide antagonizing ErbB2/Neu inhibits breast tumor growth and metastasis. Cell Rep. 2014;8(6):1714-1721. doi:10.1016/j.celrep.2014.07.044

61. Lächelt U, Wagner E. Nucleic acid therapeutics using polyplexes: a journey of 50 years (and Beyond). Chem Rev. 2015;115 (19):11043-11078. doi:10.1021/cr5006793

62. Hao F, Dong S, Yang C, et al. Targeted and efficient delivery of siRNA using tunable polymeric hybrid micelles for tumor therapy. Anticancer Res. 2019;39(3):1169-1178. doi:10.21873/ anticanres. 13226

63. Patnaik S, Gupta KC. Novel polyethylenimine-derived nanoparticles for in vivo gene delivery. Expert Opin Drug Deliv. 2013;10 (2):215-228. doi:10.1517/17425247.2013.744964

64. Xu F, Zhong H, Chang Y, et al. Targeting death receptors for drug-resistant cancer therapy: codelivery of pTRAIL and monensin using dual-targeting and stimuli-responsive self-assembling nanocomposites. Biomaterials. 2018;158:56-73. doi:10.1016/j. biomaterials.2017.12.018

65. Tan J, Wang $\mathrm{H}, \mathrm{Xu} \mathrm{F}$, et al. Poly- $\gamma$-glutamic acid-based GGTtargeting and surface camouflage strategy for improving cervical cancer gene therapy. J Mater Chem B. 2017;5(6):1315-1327. doi:10.1039/c6tb02990f

66. Choi KY, Silvestre OF, Huang X, et al. A nanoparticle formula for delivering siRNA or miRNAs to tumor cells in cell culture and in vivo. Nat Protoc. 2014;9(8):1900-1915. doi:10.1038/nprot.2014. 128

67. Saw PE, Yao H, Lin C, Tao W, Farokhzad OC, Xu X. Stimuliresponsive polymer-prodrug hybrid nanoplatform for multistage siRNA delivery and combination cancer therapy. Nano Lett. 2019;19(9):5967-5974. doi:10.1021/acs.nanolett.9b01660

68. Zhao Y, Wang W, Guo S, et al. PolyMetformin combines carrier and anticancer activities for in vivo siRNA delivery. Nat Commun. 2016;7:11822. doi:10.1038/ncomms11822

69. Augustine R, Kalva N, Kim HA, Zhang Y, Kim I. pH-responsive polypeptide-based smart nano-carriers for theranostic applications. Molecules (Basel, Switzerland). 2019;24(16):2961 doi:10.3390/molecules24162961

70. Benjaminsen RV, Mattebjerg MA, Henriksen JR, Moghimi SM, Andresen TL. The possible "proton sponge " effect of polyethylenimine (PEI) does not include change in lysosomal pH. $\mathrm{Mol}$ Ther. 2013;21(1):149-157. doi:10.1038/mt.2012.185

71. Oh JK. Disassembly and tumor-targeting drug delivery of reduction-responsive degradable block copolymer nanoassemblies. Polym Chem. 2019;10(13):1554-1568. doi:10.10 39/c8py01808a
72. Karimi M, Ghasemi A, Sahandi Zangabad P, et al. Smart micro/ nanoparticles in stimulus-responsive drug/gene delivery systems. Chem Soc Rev. 2016;45(5):1457-1501. doi:10.1039/c5cs00798d

73. Ataullakhanov FI, Vitvitsky VM. What determines the intracellular ATP concentration. Biosci Rep. 2002;22(5-6):501-511. doi:10.1023/a:1022069718709

74. Wu Y, Zhang $\mathrm{X}$, Li $\mathrm{H}$, et al. A core/shell stabilized polysaccharide-based nanoparticle with intracellular environment-sensitive drug delivery for breast cancer therapy. J Mater Chem B. 2018;6(41):6646-6659. doi:10.1039/c8tb00633d

75. Liu KS, Liu H, Qi JH, et al. SNX-2112, an Hsp90 inhibitor, induces apoptosis and autophagy via degradation of Hsp90 client proteins in human melanoma A-375 cells. Cancer Lett. 2012;318 (2):180-188. doi:10.1016/j.canlet.2011.12.015

76. Lu HH, Huang CH, Shiue TY, et al. Highly efficient gene release in spatiotemporal precision approached by light and $\mathrm{pH}$ dual responsive copolymers. Chem Sci. 2019;10(1):284-292. doi:10. 1039/c8sc01494a

77. He H, Zhu R, Sun W, Cai K, Chen Y, Yin L. Selective cancer treatment via photodynamic sensitization of hypoxia-responsive drug delivery. Nanoscale. 2018;10(6):2856-2865. doi:10.1039/ c7nr07677k

78. Tiwari AP, Hwang TI, Oh JM, et al. pH/NIR-responsive polypyrrole-functionalized fibrous localized drug-delivery platform for synergistic cancer therapy. ACS Appl Mater Interfaces. 2018;10(24):20256-20270. doi:10.1021/acsami.7b17 664

79. Zeng $\mathrm{X}$, Wang $\mathrm{Y}$, Han J, et al. Fighting against drug-resistant tumors using a dual-responsive $\mathrm{Pt}(\mathrm{IV}) / \mathrm{Ru}(\mathrm{II})$ bimetallic polymer. Adv Mater. 2020:e2004766. doi:10.1002/adma.20200 4766.

80. Zhou J, Han Y, Yang Y, et al. Phospholipid-decorated glycogen nanoparticles for stimuli-responsive drug release and synergetic chemophotothermal therapy of hepatocellular carcinoma. ACS Appl Mater Interfaces. 2020;12(20):23311-23322. doi:10.1021/ acsami.0c02785

81. Chen W, Achazi K, Schade B, Haag R. Charge-conversional and reduction-sensitive poly(vinyl alcohol) nanogels for enhanced cell uptake and efficient intracellular doxorubicin release. J Control Release. 2015;205:15-24. doi:10.1016/j. jconrel.2014.11.012

82. Guo Z, Sui J, Ma M, et al. pH-Responsive charge switchable PEGylated $\varepsilon$-poly-1-lysine polymeric nanoparticles-assisted combination therapy for improving breast cancer treatment. J Control Release. 2020;326:350-364. doi:10.1016/j.jconrel.20 20.07.030

83. Yoo J, Sanoj Rejinold N, Lee D, Jon S, Kim YC. Proteaseactivatable cell-penetrating peptide possessing ROS-triggered phase transition for enhanced cancer therapy. J Control Release. 2017;264:89-101. doi:10.1016/j.jconrel.2017.08.026

84. Li Y, Xu X, Zhang X, Li Y, Zhang Z, Gu Z. Tumor-specific multiple stimuli-activated dendrimeric nanoassemblies with metabolic blockade surmount chemotherapy resistance. ACS Nano. 2017;11(1):416-429. doi:10.1021/acsnano.6b06161

85. Han H, Valdepérez D, Jin Q, et al. Dual enzymatic reaction-assisted gemcitabine delivery systems for programmed pancreatic cancer therapy. ACS Nano. 2017;11(2):1281-1291. doi:10.1021/acsnano.6b05541

86. Gao Y, Jia L, Wang Q, et al. pH/Redox dual-responsive polyplex with effective endosomal escape for codelivery of siRNA and doxorubicin against drug-resistant cancer cells. ACS Appl Mater Interfaces. 2019;11(18):16296-16310. doi:10.1021/acsami.9b02016

87. Hyun LJ, Woo CH, Joon MS, Geun CB. Dual-stimuli responsive mesoporous copper (II) sulfide nanocomposite for chemo-photothermal synergistic therapy. Micropor Mesopor Mater. 2020;302. doi:10.1016/j.micromeso.2020.110228. 
88. Yin $\mathrm{W}, \mathrm{Ke} \mathrm{W}, \mathrm{Lu} \mathrm{N}$, et al. Glutathione and reactive oxygen species dual-responsive block copolymer prodrugs for boosting tumor site-specific drug release and enhanced antitumor efficacy. Biomacromolecules. 2020;21(2):921-929. doi:10.1021/ acs.biomac.9b01578

89. Mondal B, Pandey B, Parekh N, et al. Amphiphilic mannose-6-phosphate glycopolypeptide-based bioactive and responsive self-assembled nanostructures for controlled and targeted lysosomal cargo delivery. Biomater Sci. 2020;8(22):63 22-6336. doi:10.1039/d0bm01469a

90. Han X, Wang L, Du J, Dou J, Yuan J, Shen J. Keratin-dopamine conjugate nanoparticles as $\mathrm{pH} / \mathrm{GSH}$ dual responsive drug carriers. $J$ Biomater Sci Polym Ed. 2020;1-13. doi:10.1080/092050 63.2020 .1803182

91. Song Y, Cai L, Tian Z, Wu Y, Chen J. Phytochemical curcumin-coformulated, silver-decorated melanin-like polydopamine/mesoporous silica composites with improved antibacterial and chemotherapeutic effects against drug-resistant cancer cells. ACS Omega. 2020;5(25):15083-15094. doi:10.1021/acsomega.0c00912

92. Li Y, Hou H, Zhang P, Zhang Z. Co-delivery of doxorubicin and paclitaxel by reduction/ $\mathrm{pH}$ dual responsive nanocarriers for osteosarcoma therapy. Drug Deliv. 2020;27(1):1044-1053. doi:10.10 80/10717544.2020.1785049

93. Su M, Xiao S, Shu M, et al. Enzymatic multifunctional biodegradable polymers for $\mathrm{pH}$ - and ROS-responsive anticancer drug delivery. Colloids Surf B Biointerfaces. 2020;193:111067. doi:10. 1016/j.colsurfb.2020.111067

94. Jiang D, Mu W, Pang X, et al. Cascade cytosol delivery of dual-sensitive micelle-tailored vaccine for enhancing cancer immunotherapy. ACS Appl Mater Interfaces. 2018;10(44):37 797-37811. doi:10.1021/acsami.8b09946

95. Kim J, Lee YM, Kim H, Park D, Kim J, Kim WJ. Phenylboronic acid-sugar grafted polymer architecture as a dual stimuli-responsive gene carrier for targeted anti-angiogenic tumor therapy. Biomaterials. 2016;75:102-111. doi:10.1016/j. biomaterials.2015.10.022

96. Zhao Y, Shi C, Yang X, et al. $\mathrm{pH}$ - and temperature-sensitive hydrogel nanoparticles with dual photoluminescence for bioprobes. ACS Nano. 2016;10(6):5856-5863. doi:10.1021/acsnano.6b00770

97. Lu N, Huang P, Fan W, et al. Tri-stimuli-responsive biodegradable theranostics for mild hyperthermia enhanced chemotherapy. Biomaterials. 2017;126:39-48. doi:10.1016/j.biomaterials.2017. 02.025

98. Zhang L, Sheng D, Wang D, et al. Bioinspired multifunctional melanin-based nanoliposome for photoacoustic/magnetic resonance imaging-guided efficient photothermal ablation of cancer. Theranostics. 2018;8(6):1591-1606. doi:10.7150/thno.22430

99. Feng T, Zhou L, Wang Z, et al. Dual-stimuli responsive nanotheranostics for mild hyperthermia enhanced inhibition of Wnt/ $\beta$ catenin signaling. Biomaterials. 2020;232:119709. doi:10.1016/j. biomaterials.2019.119709

100. Wu S, Butt HJ. Near-infrared-sensitive materials based on upconverting nanoparticles. Adv Mater. 2016;28(6):1208-1226. doi:10.1002/adma.201502843

101. Zhang H, Jiao X, Chen Q, et al. A multi-functional nanoplatform for tumor synergistic phototherapy. Nanotechnology. 2016;27 (8):085104. doi:10.1088/0957-4484/27/8/085104

102. $\mathrm{Xu} \mathrm{J,} \mathrm{Kuang} \mathrm{Y,} \mathrm{Lv} \mathrm{R,} \mathrm{et} \mathrm{al.} \mathrm{Charge} \mathrm{convertibility} \mathrm{and} \mathrm{near}$ infrared photon co-enhanced cisplatin chemotherapy based on upconversion nanoplatform. Biomaterials. 2017;130:42-55. doi:10.1016/j.biomaterials.2017.03.041

103. Yong Y, Zhou L, Zhang S, et al. Gadolinium polytungstate nanoclusters: a new theranostic with ultrasmall size and versatile properties for dual-modal MR/CT imaging and photothermal therapy/radiotherapy of cancer. NPG Asia Mater. 2016;8(5): e273. doi:10.1038/am.2016.63
104. Yu J, Yin W, Peng T, et al. Biodistribution, excretion, and toxicity of polyethyleneimine modified $\mathrm{NaYF}(4)$ : yb, Erupconversion nanoparticles in mice via different administration routes. Nanoscale. 2017;9(13):4497-4507. doi:10.1039/c7nr00078b

105. Dong X, Yin W, Zhang X, et al. Intelligent $\operatorname{MoS}(2)$ nanotheranostic for targeted and enzyme-/pH-/NIR-responsive drug delivery to overcome cancer chemotherapy resistance guided by PET imaging. ACS Appl Mater Interfaces. 2018;10(4):4271-4284. doi:10.1021/acsami.7b17506

106. Wang S, Li X, Chen Y, et al. A facile one-pot synthesis of a two-dimensional MoS2/Bi2S3 composite theranostic nanosystem for multi-modality tumor imaging and therapy. Adv Mater. 2015;27(17):2775-2782. doi:10.1002/adma.201500870

107. Zhao X, Yang CX, Chen LG, Yan XP. Dual-stimuli responsive and reversibly activatable theranostic nanoprobe for precision tumor-targeting and fluorescence-guided photothermal therapy. Nat Commun. 2017;8:14998. doi:10.1038/ncomms14998

108. Poulose AC, Veeranarayanan S, Mohamed MS, et al. Multistimuli responsive $\mathrm{Cu} 2 \mathrm{~S}$ nanocrystals as trimodal imaging and synergistic chemo-photothermal therapy agents. Nanoscale. 2015;7(18):8378-8388. doi:10.1039/c4nr07139e

109. Cao H, Yang Y, Chen X, Shao Z. Intelligent Janus nanoparticles for intracellular real-time monitoring of dual drug release. Nanoscale. 2016;8(12):6754-6760. doi:10.1039/c6nr00987e

110. Lai J, Shah BP, Garfunkel E, Lee KB. Versatile fluorescence resonance energy transfer-based mesoporous silica nanoparticles for real-time monitoring of drug release. ACS Nano. 2013;7 (3):2741-2750. doi:10.1021/nn400199t

111. Huang J, Zong C, Shen H, Cao Y, Ren B, Zhang Z. Tracking the intracellular drug release from graphene oxide using surface-enhanced Raman spectroscopy. Nanoscale. 2013;5 (21):10591-10598. doi:10.1039/c3nr03264g

112. Kim H, Kim J, Lee M, Choi HC, Kim WJ. Stimuli-regulated enzymatically degradable smart graphene-oxide-polymer nanocarrier facilitating photothermal gene delivery. Adv Healthcare Mater. 2016;5(15):1918-1930. doi:10.1002/adhm.201600246

113. Ye S, Wang F, Fan Z, et al. Light/pH-triggered biomimetic red blood cell membranes camouflaged small molecular drug assemblies for imaging-guided combinational chemo-photothermal therapy. ACS Appl Mater Interfaces. 2019;11(17):15262-15275. doi:10.1021/acsami.9b00897

114. Ao L, Wu C, Liu K, et al. Polydopamine-derivated hierarchical nanoplatforms for efficient dual-modal imaging-guided combination in vivo cancer therapy. ACS Appl Mater Interfaces. 2018;10 (15):12544-12552. doi:10.1021/acsami.8b02973

115. Hayashi K, Maruhashi T, Nakamura M, Sakamoto W, Yogo T. One-pot synthesis of dual stimulus-responsive degradable hollow hybrid nanoparticles for image-guided trimodal therapy. $A d v$ Funct Mater. 2016;26(47):8613-8622. doi:10.1002/adfm.20160 3394

116. Fang S, Lin J, Li C, et al. Dual-stimuli responsive nanotheranostics for multimodal imaging guided trimodal synergistic therapy. Small. 2017;13(6). doi:10.1002/smll.201602580

117. Zhou Z, Hu K, Ma R, et al. Dendritic platinum-copper alloy nanoparticles as theranostic agents for multimodal imaging and combined chemophotothermal therapy. Adv Funct Mater. 2016;26 (33):5971-5978. doi:10.1002/adfm.201601754

118. Lin X, Song X, Zhang Y, et al. Multifunctional theranostic nanosystems enabling photothermal-chemo combination therapy of triple-stimuli-responsive drug release with magnetic resonance imaging. Biomater Sci. 2020;8(7):1875-1884. doi:10.1039/c9bm $01482 \mathrm{a}$

119. Sun S, Chen Q, Tang Z, et al. Tumor microenvironment stimuli-responsive fluorescence imaging and synergistic cancer therapy by Carbon-Dot-Cu nanoassemblies. Angew Chem. 2020. doi:10.1002/anie.202007786 
120. Massoumi B, Farnudiyan-Habibi A, Derakhshankhah H, et al. theranosticA novel multi-stimuli-responsive nanomedicine based on FeO@Au nanoparticles against cancer. Drug Dev Ind Pharm. 2020:1-12. doi:10.1080/03639045.2020.1821052.

121. Gou S, Yang J, Ma Y, et al. Multi-responsive nanococktails with programmable targeting capacity for imaging-guided mitochondrial phototherapy combined with chemotherapy. J Control Release. 2020;327:371-383. doi:10.1016/j.jconrel.2020.08.014

122. Kuang Y, Zhang Y, Zhao Y, et al. Dual-stimuli-responsive multifunctional GdHfO nanoparticles for MRI-guided combined chemo-/photothermal-/radiotherapy of resistant tumors. ACS Appl Mater Interfaces. 2020;12(32):35928-35939. doi:10.1021/ acsami.0c09422

123. Kang S, Baskaran R, Ozlu B, et al. T-positive Mn-doped multi-stimuli responsive poly(L-DOPA) nanoparticles for photothermal and photodynamic combination cancer therapy. Biomedicines. 2020;8(10):417. doi:10.3390/biomedicines 8100 417

124. Wang J, Chang $\mathrm{Y}$, Luo $\mathrm{H}$, et al. Designing immunogenic nanotherapeutics for photothermal-triggered immunotherapy involving reprogramming immunosuppression and activating systemic antitumor responses. Biomaterials. 2020;255. doi:10.1016/ j.biomaterials.2020.120153

125. Topalian SL, Taube JM, Anders RA, Pardoll DM. Mechanismdriven biomarkers to guide immune checkpoint blockade in cancer therapy. Nat Rev Cancer. 2016;16(5):275-287. doi:10.1038/ nrc. 2016.36

126. Sau S, Alsaab HO, Bhise K, Alzhrani R, Nabil G, Iyer AK. Multifunctional nanoparticles for cancer immunotherapy: a groundbreaking approach for reprogramming malfunctioned tumor environment. J Control Release. 2018;274:24-34. doi:10. 1016/j.jconrel.2018.01.028

127. Selvan SR, Dowling JP, Kelly WK, Lin J. Indoleamine 2,3-dioxygenase (IDO): biology and target in cancer immunotherapies. Curr Cancer Drug Targets. 2016;16 (9):755-764. doi:10.2174/1568009615666151030102250

128. Cheng K, Ding Y, Zhao Y, et al. Sequentially responsive therapeutic peptide assembling nanoparticles for dual-targeted cancer immunotherapy. Nano Lett. 2018;18(5):3250-3258. doi:10.1021/ acs.nanolett.8b01071

129. Ding Y, Ji T, Zhao Y, et al. Improvement of stability and efficacy of $\mathrm{C} 16 \mathrm{Y}$ therapeutic peptide via molecular self-assembly into tumor-responsive nanoformulation. Mol Cancer Ther. 2015;14 (10):2390-2400. doi:10.1158/1535-7163.Mct-15-0484

130. Chen DS, Mellman I. Elements of cancer immunity and the cancer-immune set point. Nature. 2017;541(7637):321-330. doi:10.1038/nature21349

131. Feng B, Zhou F, Hou B, et al. Binary cooperative prodrug nanoparticles improve immunotherapy by synergistically modulating immune tumor microenvironment. Adv Mater. 2018;30(38). doi:10.1002/adma.201803001

132. Zheng D-W, Chen J-L, Zhu J-Y, et al. Highly integrated nano-platform for breaking the barrier between chemotherapy and immunotherapy. Nano Lett. 2016;16(7):4341-4347. doi:10.10 21/acs.nanolett.6b01432
133. Hou B, Zhou L, Wang H, et al. Engineering stimuli-activatable boolean logic prodrug nanoparticles for combination cancer immunotherapy. Adv Mater. 2020;32(12):e1907210. doi:10.1002/ adma.201907210

134. Huynh E, Leung BY, Helfield BL, et al. In situ conversion of porphyrin microbubbles to nanoparticles for multimodality imaging. Nat Nanotechnol. 2015;10(4):325-332. doi:10.1038/ nnano. 2015.25

135. Kawai T, Akira S. Toll-like receptors and their crosstalk with other innate receptors in infection and immunity. Immunity. 2011;34(5):637-650. doi:10.1016/j.immuni.2011.05.006

136. Yin T, He S, Wang Y. Toll-like receptor 7/8 agonist, R848, exhibits antitumoral effects in a breast cancer model. Mol Med Rep. 2015;12(3):3515-3520. doi:10.3892/mmr.2015.3885

137. Liu Y, Qiao L, Zhang S, et al. Dual $\mathrm{pH}$-responsive multifunctional nanoparticles for targeted treatment of breast cancer by combining immunotherapy and chemotherapy. Acta Biomater. 2018;66:310-324. doi:10.1016/j.actbio.2017.11.010

138. $\mathrm{Hu} \mathrm{Q}$, Chen $\mathrm{Q}, \mathrm{Gu} \mathrm{Z}$. Advances in transformable drug delivery systems. Biomaterials. 2018;178:546-558. doi:10.1016/j. biomaterials.2018.03.056

139. Safra T, Muggia F, Jeffers S, et al. Pegylated liposomal doxorubicin (doxil): reduced clinical cardiotoxicity in patients reaching or exceeding cumulative doses of $500 \mathrm{mg}$ / m(2). Ann Oncol. 2000;11(8):1029-1033. doi:10.1023/a:10 08365716693

140. Blanco E, Shen H, Ferrari M. Principles of nanoparticle design for overcoming biological barriers to drug delivery. Nat Biotechnol. 2015;33(9):941-951. doi:10.1038/nbt.3330

141. Yan L, Shen J, Wang J, Yang X, Dong S, Lu S. Nanoparticlebased drug delivery system: a patient-friendly chemotherapy for oncology. Dose Response. 2020;18(3):1559325820936161. doi:10.1177/1559325820936161

142. Barnes LD, Giuliano EA, Ota J. Cellular localization of Visudyne as a function of time after local injection in an in vivo model of squamous cell carcinoma: an investigation into tumor cell death. Vet Ophthalmol. 2010;13(3):158-165. doi:10.1111/j.14635224.2010.00775.x

143. Caster JM, Patel AN, Zhang T, Wang A. Investigational nanomedicines in 2016: a review of nanotherapeutics currently undergoing clinical trials. Wiley Interdiscip Rev Nanomed Nanobiotechnol. 2017;9(1):e1416. doi:10.1002/wnan.1416

144. Liu D, Yang F, Xiong F, Gu N. The smart drug delivery system and its clinical potential. Theranostics. 2016;6(9):1306-1323 doi:10.7150/thno. 14858

145. Ventola CL. Progress in nanomedicine: approved and investigational nanodrugs. P T. 2017;42(12):742-755.

146. Du J, Lane LA, Nie S. Stimuli-responsive nanoparticles for targeting the tumor microenvironment. $J$ Control Release. 2015;219:205-214. doi:10.1016/j.jconrel.2015.08.050

147. Dai Y, Xu C, Sun X, Chen X. Nanoparticle design strategies for enhanced anticancer therapy by exploiting the tumour microenvironment. Chem Soc Rev. 2017;46(12):3830-3852. doi:10.1039/c6cs00592f
International Journal of Nanomedicine

\section{Publish your work in this journal}

The International Journal of Nanomedicine is an international, peerreviewed journal focusing on the application of nanotechnology in diagnostics, therapeutics, and drug delivery systems throughout the biomedical field. This journal is indexed on PubMed Central, MedLine, CAS, SciSearch ${ }^{\mathbb{R}}$, Current Contents ${ }^{\mathbb{B}} /$ Clinical Medicine, $^{2}$
Journal Citation Reports/Science Edition, EMBase, Scopus and the Elsevier Bibliographic databases. The manuscript management system is completely online and includes a very quick and fair peer-review system, which is all easy to use. Visit http://www.dovepress.com/ testimonials.php to read real quotes from published authors. 